\title{
Single-cell analysis reveals cellular heterogeneity and molecular determinants of
}

\section{hypothalamic leptin-receptor cells}

N. Kakava-Georgiadou ${ }^{1,4}$, J.F. Severens ${ }^{1,4}$, A.M. Jørgensen ${ }^{3}$, K.M. Garner ${ }^{1}$, M.C.M Luijendijk ${ }^{1}$, V. Drkelic ${ }^{1}$, R. van Dijk ${ }^{1}$, T.H. Pers ${ }^{3}$, O. Basak ${ }^{1,5,{ }^{*}}$, R.A.H. Adan ${ }^{1,2,5,{ }^{*}}$

1 Department of Translational Neuroscience, Division of Neuroscience, Brain Center Rudolf Magnus, University Medical Center Utrecht, Utrecht University, The Netherlands

2 Institute of Neuroscience and Physiology, The Sahlgrenska Academy at the University of Gothenburg, Sweden

3 Novo Nordisk Foundation Centre for Basic Metabolic Research, Faculty of Health and Medical Sciences, University of Copenhagen, Copenhagen, Denmark

4 shared first authors

5 shared senior authors

*Corresponding authors: r.a.h.adan@umcutrecht.nl and o.basak@umcutrecht.nl

\section{Abstract}

Hypothalamic nuclei which regulate homeostatic functions express leptin receptor (LepR), the primary target of the satiety hormone leptin. Single-cell RNA sequencing (scRNA-seq) has facilitated the discovery of a variety of hypothalamic cell types. However, low abundance of LepR transcripts prevented further characterization of LepR cells. Therefore, we perform scRNA-seq on isolated LepR cells and identify eight neuronal clusters, including three uncharacterized Trh-expressing populations as well as 17 non-neuronal populations including tanycytes, oligodendrocytes and endothelial cells. Food restriction had a major impact on Agrp neurons and changed the expression of obesity-associated genes. Multiple cell clusters were enriched for GWAS signals of obesity. We further explored changes in the gene regulatory landscape of LepR cell types. We thus reveal the molecular signature of distinct populations with diverse 
bioRxiv preprint doi: https://doi.org/10.1101/2020.07.23.217729; this version posted July 24, 2020. The copyright holder for this preprint (which was not certified by peer review) is the author/funder, who has granted bioRxiv a license to display the preprint in perpetuity. It is made available under aCC-BY-NC-ND 4.0 International license.

neurochemical profiles, which will aid efforts to illuminate the multi-functional nature of leptin's action in the hypothalamus. 


\section{Introduction}

Leptin is a hormone predominantly produced by white adipose tissue and secreted into the blood circulation ${ }^{1}$. Leptin regulates metabolism and appetite by inhibiting food intake, lowering body weight and increasing metabolic rate ${ }^{2}$. Circulating leptin levels are proportional to body fat amount and body mass index $(\mathrm{BMI})^{3}$. Leptin also responds to acute changes in energy balance: fasting decreases and feeding increases leptin levels ${ }^{4}$. Obese people have high circulating leptin levels suggesting decreased sensitivity to leptin ${ }^{3,4}$, which is referred to as leptin resistance.

The hypothalamus plays a major role in the effects of leptin on energy balance, with strong leptin receptor (LepR) expression in the Arcuate nucleus ( $\operatorname{Arc})$. The two most well studied LepR neurons in the Arc are the orexigenic AGRP/NPY and the anorexigenic POMC/CART neurons. Leptin inhibits Agrp and stimulates Pomc neurons ${ }^{5-7}$, while Agrp neurons are the most sensitive hypothalamic cell population to energy deficit ${ }^{8-10}$. LepR is also expressed in less defined neurons in the ventromedial hypothalamus (VMH), dorsomedial hypothalamus (DMH), preoptic area (PO), pre-mammillary nucleus (PMv), lateral hypothalamus (LH) and weakly in the paraventricular nucleus (PVN) $)^{11-13}$, that contribute to leptin's effects on homeostatic functions ${ }^{14-16}$. Besides neuronal cells, LepR expression has also been reported in astrocytes ${ }^{17,18}$, tanycytes ${ }^{19}$, endothelial cells ${ }^{20}$, microglia and macrophages ${ }^{21}$, oligodendrocytes ${ }^{22}$ and vascular leptomeningeal cells (VLMCs) ${ }^{23}$.

Thus, the hypothalamic LepR population of cells is highly diverse, but a full description of the diversity of their transcriptomes remains unresolved. Unravelling the transcriptome of hypothalamic leptin receptor expressing cells helps to identify cell-specific targets to treat obesity. In the mouse hypothalamus, singlecell RNA-seq has provided valuable information about the molecular profile of hypothalamic cell types and new neuronal and non-neuronal subtypes ${ }^{9,10,24}$. However, in hypothalamic scRNAseq datasets it is difficult to identify LepR cells due to their scarcity and the low abundance of LepR transcripts.

To overcome these hurdles, we took a biased approach to identify all LepR-expressing cells in the hypothalamus. We used a LepR-Cre mouse crossed with a mouse expressing the Cre-dependent fluorescent protein tdTomato to specifically isolate hypothalamic LepR cells using FACS and sequence their transcriptome at single-cell resolution. We identify new LepR subtypes, define their molecular signatures, active gene regulatory networks and their enrichment in GWAS Body Mass index signals. Furthermore, we show that fasting induces major transcriptional changes in Agrp LepR neurons. Overall, our dataset provides insight into the molecular makeup of hypothalamic LepR cells and a source for future studies. 


\section{Results}

To characterize LepR $^{+}$hypothalamic cells, we optimized isolation of intact single cells from the hypothalamus of LepR-tdTomato mice (see Methods). The tdTomato-expressing cells from fed or $24 \mathrm{~h}-$ fasted animals were FACS-sorted based on tdTomato fluorescence (Fig. 1a, S1a) followed by single-cell RNA sequencing (scRNAseq, see Methods).

A total of 1,288 tdTomato ${ }^{+}$cells were sorted in four plates/batches (Fig. S1b) and were sequenced with Cel-Seq $2^{25}$. Of all tdTomato cells, 1,048 cells were from fed animals ("fed cells") and 240 cells from fasted animals ("fasted cells") and were subsequently used for analysis using Scanpy ${ }^{26}$. Cells containing less than 2,000 genes and with more than 50\% of the reads belonging to ERCC spike-in RNA (indicative of poor caption of mRNA from cells) or with more than 15\% mitochondrial gene expression (indicative of RNA degradation and poor cell viability) were excluded (Fig.S1c). These steps left 824 cells for analysis, 692 cells from the fed condition and 132 cells from the fasted condition (Fig. S1b), with a high-quality dataset containing a median of 12,082 unique counts and 4,756 genes per cell. To overcome the effect of sequencing depth between neurons and non-neuronal cells in clustering, we used downsampling prior to the analysis (see Methods for details).

Unsupervised clustering using the graph - based Leiden algorithm identified a total of 25 clusters (Fig. 1b), which were further assigned to cell types based on expression of established cell type markers (Fig. 1c). Hierarchical clustering indicated a clear separation of neuronal and non-neuronal clusters (Fig. S2a). Eight neuronal clusters with 373 cells were identified based on the expression of both Tubb3 (class III $\beta$-tubulin) and Mapt, whereas 451 cells belonged to 17 non-neuronal clusters, as defined based on the absence of both markers (Fig. 1c). We also identified a cluster of pituitary cells using Tshb as a marker and excluded it from further analysis. The high complexity of our scRNAseq dataset allows us to detect rarely expressed genes in specific cell types, which are notoriously hard to detect with single cell methods. As expected, Lepr mRNA was not identified in all cells, due to the low abundance of LepR transcripts, but it was most prominently expressed in Agrp, Trh_Th and Vascular leptomeningeal cells (VLMC) clusters (Fig. S2b). 
bioRxiv preprint doi: https://doi.org/10.1101/2020.07.23.217729; this version posted July 24, 2020. The copyright holder for this preprint (which was not certified by peer review) is the author/funder, who has granted bioRxiv a license to display the preprint in perpetuity. It is made available under aCC-BY-NC-ND 4.0 International license.

\section{Figure 1}
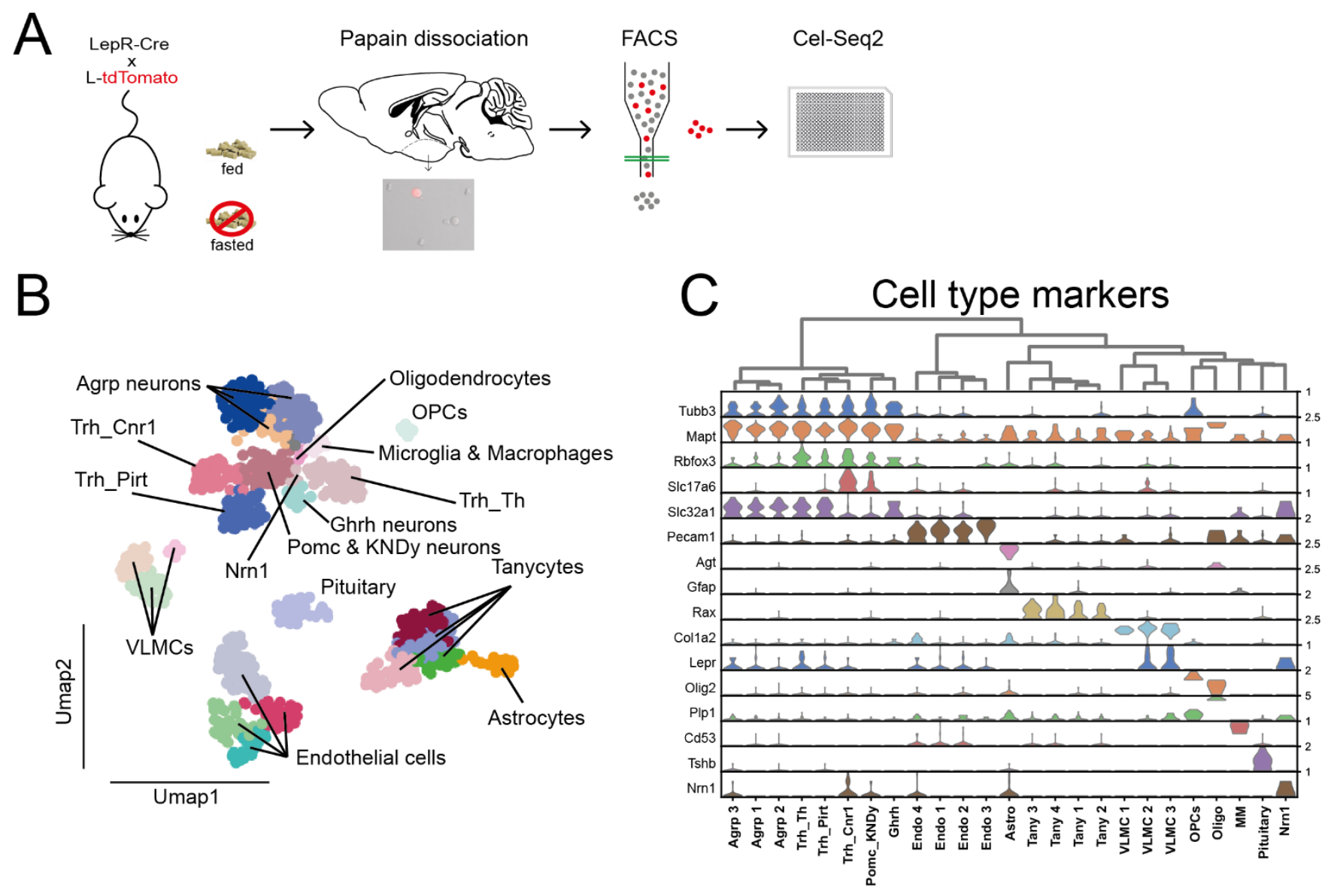


\section{Neuronal cells}

Eight neuronal ( $\mathrm{Tubb3}^{+} \mathrm{Mapt}^{+}$) clusters were identified and assigned to subtypes based on known or novel marker genes (Fig. 1b, c and Fig. 2a, b). Based on established marker genes, the classification revealed three Agrp neuron clusters with high Agrp and Npy expression, one Pomc_KNDy neuron cluster with high expression of Pomc, Cartpt, Kiss1, Pdyn and Tac2 as well as one Ghrh neuron cluster with high Ghrh and $\mathrm{Gal}$ expression. The remaining three clusters could not be assigned based on unique genes; therefore, they were designated based on expression of Trh as well as genes that strongly marked the cluster: Trh_Pirt, Trh_Cnr1 and Trh_Th (Fig. 1c, 2a, 2b).

Based on the expression of SIC32a1 (which codes for VGAT) and SIc17a6 (which codes for VGLUT2) (Fig. 2b, 2c), Agrp, Ghrh and Trh_Th clusters were GABAergic, while the Pomc_KNDy neuron cluster contained both GABAergic and glutamatergic cells in line with the literature ${ }^{27-29}$. The Trh_Pirt and Trh_Cnr1 clusters were comprised of mostly GABAergic cells with a few glutamatergic cells.

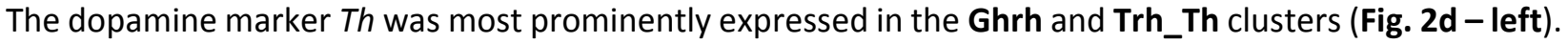
The dopaminergic marker $D d c$ was also expressed in the Ghrh cluster but mostly marked the Trh_Pirt and Trh_Cnr1 clusters (Fig. 2d - right). Besides the Pomc_KNDy cluster, high expression of Cartpt was also found in the Trh_Th and Trh_Pirt clusters (Fig. 2b). In line with what is known about LepR neurons, neither Hcrt nor Pmch were expressed in our dataset (not shown). Few cells expressing Crhr2 were found in clusters Trh_Pirt, Trh_Cnr1 and Trh_Th, while most cells with Crhr1 expression were found in Agrp clusters (Fig. 2e).

Moreover, the serotonin receptor gene Htr2c, a receptor widely distributed in hypothalamic LepR cells and a drug target of the obesity drug Lorcaserin ${ }^{30,31}$, was significantly enriched in the Trh_Cnr1 cluster, with high expression in the Trh_Pirt cluster, while it was also detected in a limited number of Pomc_KNDy cells (Fig. 2b), in which serotonin 2 c receptors have an established role in energy homeostasis ${ }^{32,33}$. Interestingly, Campbell et al. ${ }^{10}$ observed high expression of Lepr in a distinct POMC subset that was negative for Htr2c. Esr1 (Estrogen receptor 1) has an important role in metabolic regulation ${ }^{34}$ and was highly expressed in the POMC_KNDy and Ghrh clusters (Fig. 2b). A full list of expression profiles of neuropeptides and their receptors is provided in the supplementary figures (Fig. S3a, b). 


\section{Figure 2}

A

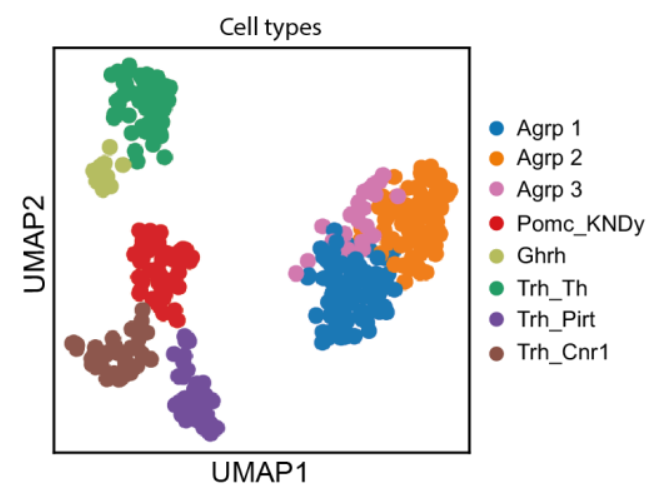

C

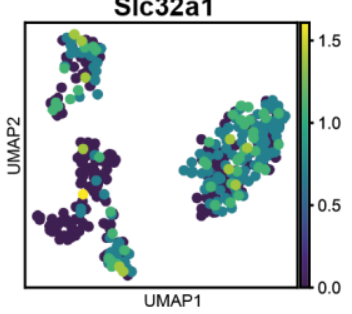

D

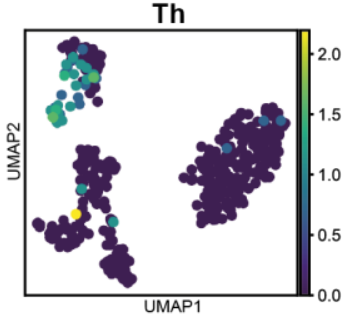

E

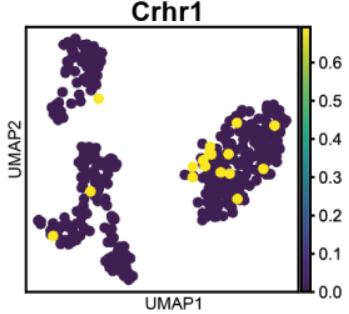

F

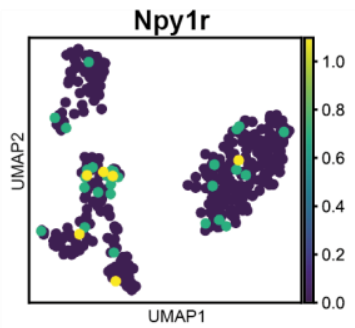

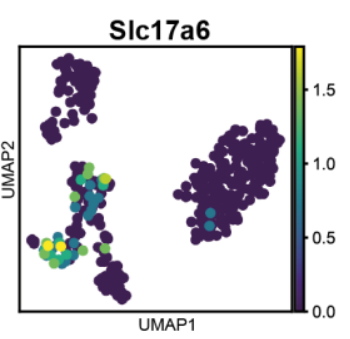

Ddc

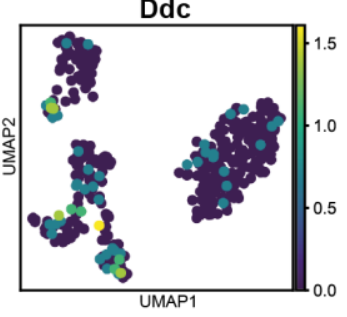

Crhr2
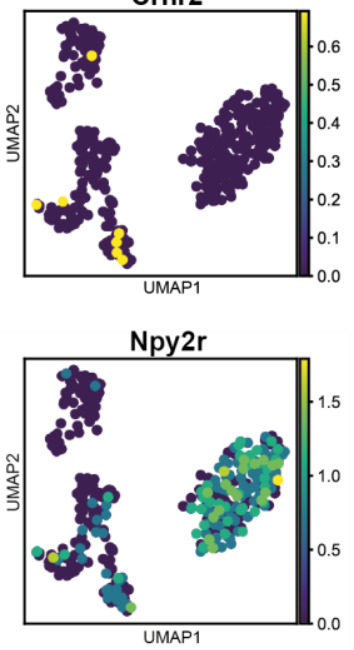

B

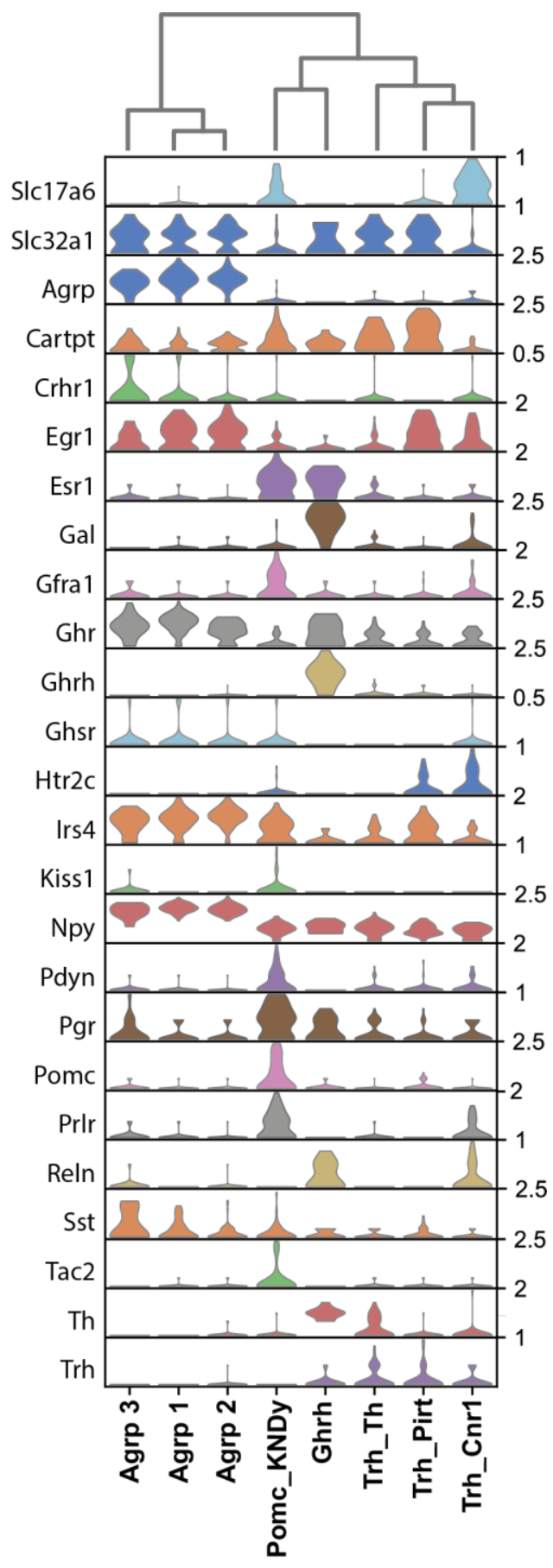


Next, we looked into the molecular signatures of specific neuronal clusters. Agrp neurons divided into 3 clusters, which all showed high expression of Ghr (growth hormone receptor), Crhr1 (corticotropinreleasing factor receptor 1), Irs4 (Insulin receptor substrate 4), Ghsr (ghrelin receptor), Egr1 (early growth response protein 1) and Sst (somatostatin) with higher enrichment in Agrp cluster 3 (Fig. 2b). As expected, the Npy2r (neuropeptide $Y$ receptor 2), a presynaptic autoreceptor, was expressed in almost all Agrp neurons $^{35}$, along with cells in the Pomc_KNDy, Trh_Pirt and Trh_Cnr1 clusters, whereas Npy1r (neuropeptide Y receptor 1) was mostly expressed in Pomc_KNDy neurons, along with other cell types (Fig. 2f).

The Pomc_KNDy cluster displayed high expression of PrIr (Prolactin receptor), Pgr (Progesterone receptor) and Gfra1 (GDNF family receptor alpha-1) (Fig. 2b).The Ghrh neuron cluster showed enrichment of Gal (Galanin) and Reln (Reelin), both of which were also expressed in lower levels in different subsets of cells in cluster Trh_Cnr1 (Fig. 2b). Most of the LepR GHRH neurons are located in the Arc and half of them engage in leptin-mediated pSTAT ${ }^{30}$. Somatostatin inhibits whereas leptin stimulates pulsatile GHRHmediated growth hormone release from the pituitary ${ }^{36-39}$. While Arc GHRH neurons express both Somatostatin receptors (Sstr1 and Sstr2 $)^{40}$, in our LepR Ghrh cluster we found expression of solely Sstr2 (Fig. S3b). Even though knock-out of LepR in GHRH neurons does not affect body weight ${ }^{30}$, this LepR population poses exceptional interest for further studies.

Three clusters showed expression of Trh (Fig. 3a). Trh_Th neurons were GABAergic neurons that also showed high expression of Cartpt (Fig. 2b, 2d), while almost half of them expressed Th (Fig. 2 d). In this cluster, there was high expression of transcription factors $L h x 1$, Lhx1os (Fig. 3a), being co-expressed in 3 cells (Fig. 3b), as well as Kit (Fig. 3a). Receptors Ptger3 and Adra1b, expressed in the preoptic nucleus (PON) and involved in BAT thermogenesis ${ }^{41-43}$ were also highly expressed (Fig. 3a, 3b). Many cells in this cluster also expressed Cxc/12 (Fig. 3a), a chemokine mainly expressed in the PVH, LH and lower in Arc, with its mRNA levels increasing upon high-fat diet in these nuclei ${ }^{44}$.

High Th expression was observed in both the Ghrh and Trh_Th clusters, with high expression of Ghrh and $\mathrm{Gal}$ in the former and low or absent expression of these markers in the latter. We therefore investigated the expression patterns of Th, Ghrh and Gal in LepR neurons in the hypothalamus, by performing IHC and FISH on hypothalamic tissue from LepR-Cre x L-tdTomato mice. Ghrh mRNA was found to be co-expressed with tdTomato mostly in the Arc and to a lesser extent in the VMH (Fig. 3d), confirming the Arc as a major co-localization site ${ }^{30}$, while Gal mRNA co-localized with tdTomato in the Arc (Fig. 3e - top), DMH and LH (Fig. 3e - bottom). Th signal was found in tdTomato-positive cells in the Arc, DMH and LH (Fig. 3f). This supports that Ghrh ${ }^{+} \mathrm{Gal}^{+} \mathrm{Th}^{+} \mathrm{LepR}^{+}$neurons are located in the Arc, while Trh_Th LepR neurons are a distinct 
population of neurons potentially located in the Arc, DMH, PVH, PON and LH. Of note, Campbell et al. ${ }^{10}$, 2017 who performed scRNAseq on the Arc and ME, identified a leptin-sensing GABAergic cluster (n11.Trh/Cxcl12) with high expression of Trh, Kit, Ptger3 and Cxcl12, all genes that were also found in the Trh_Th cluster.

The Trh_Cnr1 cluster was best defined by high expression of Cnr1 (cannabinoid receptor 1) (Fig. 3a, c), which is strongly linked to feeding behavior ${ }^{45}$, and also displayed high expession of Prlr (Prolactin receptor). This cluster also contained small subsets of neurons expressing a diversity of neuropeptides. Few cells in this cluster expressed Bdnf, which regulates feeding and energy balance ${ }^{46-48}$. Cnr1 and Bdnf are most strongly expressed in the VMH and weaklier in other hypothalamic nuclei (but not Arc), based on Allen Brain Atlas ISH data and literature ${ }^{48-50}$.

A subset of glutamatergic neurons in the Trh_Cnr1 cluster showed expression of Tac1 (Tachykinin 1) (Fig. 3a), which has been identified as a LepR neuron subtype marker in the PMv and LH by TRAP-seq and is linked to feeding behaviour and metabolism ${ }^{51,52}$. We also found expression and co-localization between markers Gal, Nts and Crh, established as LepR markers in the $\mathrm{LH}^{51,53}$, with one GABAergic cell co-expressing all three (Fig. 3c). In our histochemical analysis above, we also find co-expression of Gal mRNA with tdTomato in the $\mathrm{DMH}$, besides the $\mathrm{LH}$. This suggests that neurons in this cluster are not centralized in a single hypothalamic nucleus, but are most likely dispersed in the $\mathrm{VMH}, \mathrm{DMH}$ and $\mathrm{LH}$ hypothalamic nuclei. Neurons in the Trh_Pirt cluster were GABAergic and showed relatively high expression of Cartpt (Fig. 2b, d) in almost all of the cells of the cluster, while few cells express Crhr2 (Fig. 2e). Few cells expressing Pirt (Phosphoinositide-interacting protein), with a role in obesity ${ }^{54}$, were exclusively found in this cluster (Fig.3a). We also found sparse cells expressing Prlh (Prolactin Releasing Hormone), Pthlh (parathyroid hormone like hormone), Retn (Resistin) and Nmu (Neuromedin U) (not shown). 
Figure 3
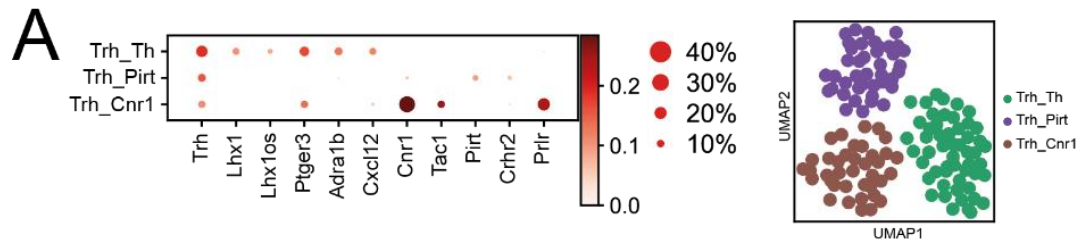

B
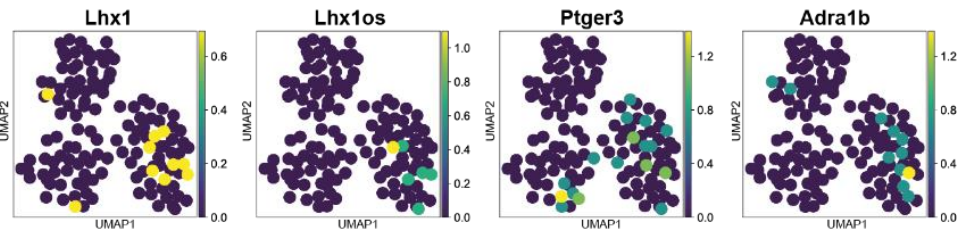

C
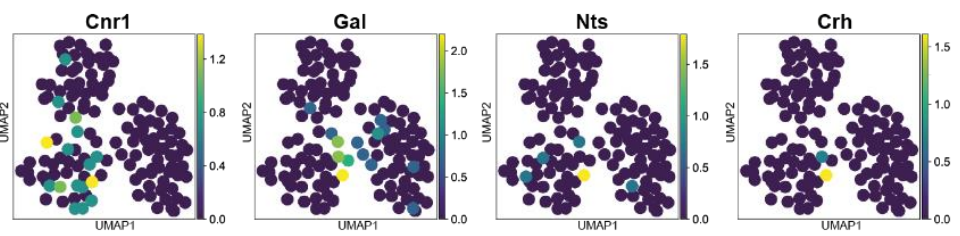

D
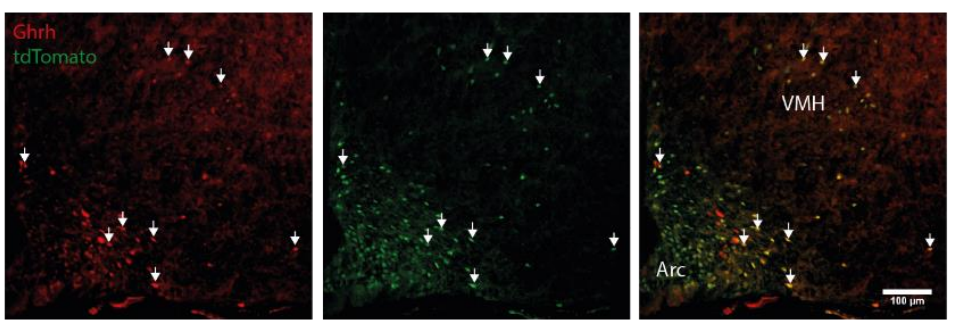

E
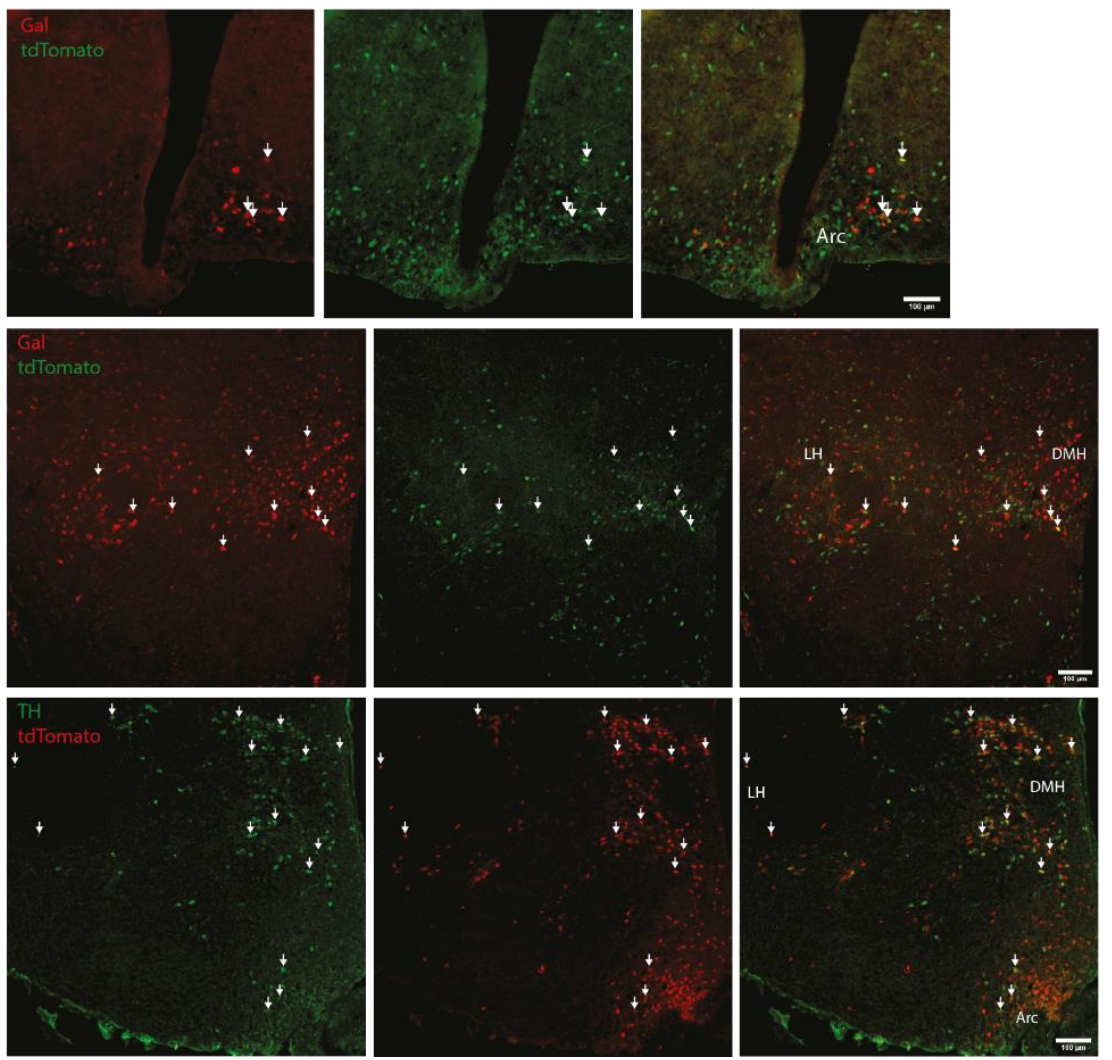

$\mathrm{F}$
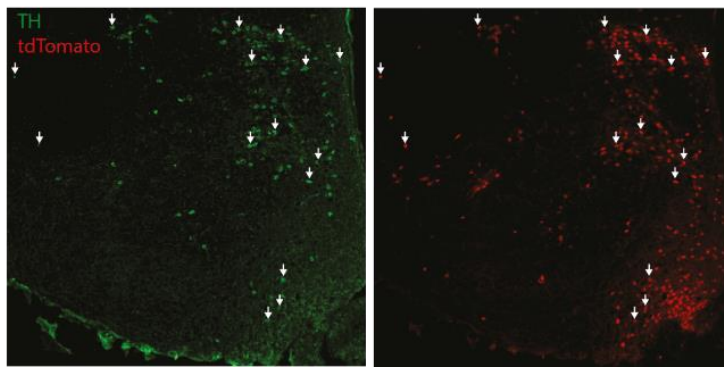


\section{Non-neuronal cells}

Seventeen (17) non-neuronal clusters, based on the absence of expression of both Tubb3 and Mapt, were assigned to subtypes based on the expression of established canonical markers. Our dataset is composed

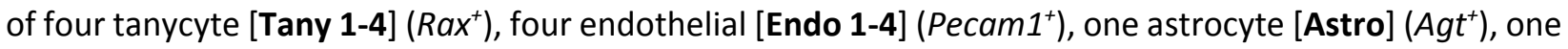
oligodendrocyte [Oligo] (Olig2 ${ }^{+}$, low Plp1), one oligodendrocyte progenitor cell [OPC] (Olig2 ${ }^{+}$, high Plp1), one microglia and macrophages [MM] $\left(\mathrm{Cd}_{5} 3^{+}\right)$, three vascular leptomeningeal cell [VLMCs 1-3] (Col1a2 $\left.{ }^{+}\right)$ clusters and one unknown cluster strongly marked by $\operatorname{Nrn1}$ (Fig. 1b, 1c).

Tanycyte clusters were almost entirely marked by Rax, Vim, Ppp1r1b, while almost half of the cells expressed Nes and only 1 cell Gfap, which is mostly found in $\alpha$ tanycyte subtypes ${ }^{55,56}$. Whereas traditionally tanycytes have been classified in 4 categories $(\alpha 1, \alpha 1, \beta 1$ and $\beta 2)$, research on their morphology and molecular signature indicates that tanycytes display great heterogeneity and they exist along a gradient ${ }^{57}$. In line with this, we did not observe a clear-cut expression of subtype-specific markers in each cluster (Fig. 4a). Tany $1 \& 2$ mostly contained $\alpha 2$ and $\beta 1$ tanycytes, since they were mostly marked by Crym and Frzb. Tany3 was mostly marked by the markers of $\beta 1$ and $\beta 2$ tanycytes Col25a1, Adm and Cacna2d2, whereas Tany4 was marked mostly by $\alpha 2$-marker Vcan as well as $\beta 2$-marker Cacna2d2. Cd59a and S/c17a8, which mark $\alpha 1$ tanycytes were expressed by very few cells. Moreover, Tany3 displayed high expression of the markers TII1 and Lrp2, which codes for Megalin and facilitates leptin transport via the BBB with transcytosis ${ }^{58,59}$ and Cntfr, confirming that in the ME there are tanycytes responsive to CTNF $^{60}$.

We identified 4 clusters of endothelial cells (Endo 1 - 4), expressing markers Pecam1, Ly6c1, Ly6a and S/co1c1 $1^{10,61}$, which form a gradient of expression, similarly to tanycytes .Endo 1 is composed mostly of venous endothelial cells due to the enriched expression of S/c38a5and $V w f$, whereas in Endo 2 S/c16a1 is highly expressed, which marks capillary cells, and Endo $\mathbf{3}$ consists of mostly arterial cells highly enriched in Vegfc, Sema3g andGkn3. Endo 4 seemed to be composed of non-endothelial cells on top of endothelial cells, due to the presence of marker genes of mural cells: Pdgfrb and Vtn for pericytes and Acta2, Myh11 for smooth muscle cells ${ }^{9,10,61}$ (Fig. 4b).

VLMC1 shows high expression of Spp1 (secreted phosphoprotein 1/Osteopontin), which mediates obesityassociated hepatic alterations ${ }^{62}$ and VLMC1 \& 2 show high expression of Igf2 (Insulin Like Growth Factor 2), whereas VLMC 3 is marked strongly by Gnrh1 (Gonadotropin Releasing Hormone 1) and Penk (Proenkephalin) (Fig. 4c).

Regarding the rest of non-neuronal clusters (Fig. 4d), we identified oligodendrocytes, mostly marked by Mobp (Myelin Associated Oligodendrocyte Basic Protein), Ermn (Ermin) and Mbp (Myelin basic protein), 
while OPCs highly expressed Fyn and Bmp4 (Bone Morphogenetic Protein 4) 9,63. Astrocytes were strongly marked by Agt (Angiotensinogen). Gfap was expressed in a small subset of astrocytes, while the rest of the cells in this cluster showed high expression of Itih3 (Fig. 4e), which has 200 fold-higher expression in mouse astrocytes compared to human ${ }^{64}$. In the Microglia and Macrophages cluster we found expression of $C \times 3 c r 1$ and Mrc1. The expression of LepR in many glial populations pinpoints leptin's important role in many different functions. Studies have shown leptin's involvement in cytokine production and activation of microglia and macrophages in the context of neuropathic pain ${ }^{65,66}$, while leptin regulates differentiation and/or myelination of oligodendrocytes ${ }^{63}$.

Several unbiased approaches profiled hypothalamus and detected a varying number of cell types. We questioned whether we could assign LepR cells identified in our study to published datasets (Fig. S4). For this, we employed Scibet which uses the E-test to predict similar cell types (see Methods, ${ }^{67}$ ). Chen et al profiled the entire hypothalamus ${ }^{9}$. LepR non-neuronal cells were easily mapped to specific non-neuronal clusters from Chen et al. (Fig. S4a). For instance, Tany 1-4 were all identified as "Tanycytes", while Endo 1-4 and VLMC 1-3 were assigned to "Epithelial 1-2". Agrp neurons 1-3 were identified as "GABA15", while Ghrh and Pomc neurons best matched GABA11 and Glu11, respectively. On the other hand, Trh neurons could not be assigned to a specific cluster, suggesting that they are either rare, share similarities with multiple clusters or could only be clustered upon sequencing at higher depth and cell numbers. Direct comparison of Lepr neurons to hypothalamic cell populations may help us determine which option holds true. Overall, we identified several subgroups of glial and neuronal cells that were originally assigned as a single cell type in other studies as well as three Trh+ cell clusters that were not reported. 


\section{Figure 4}
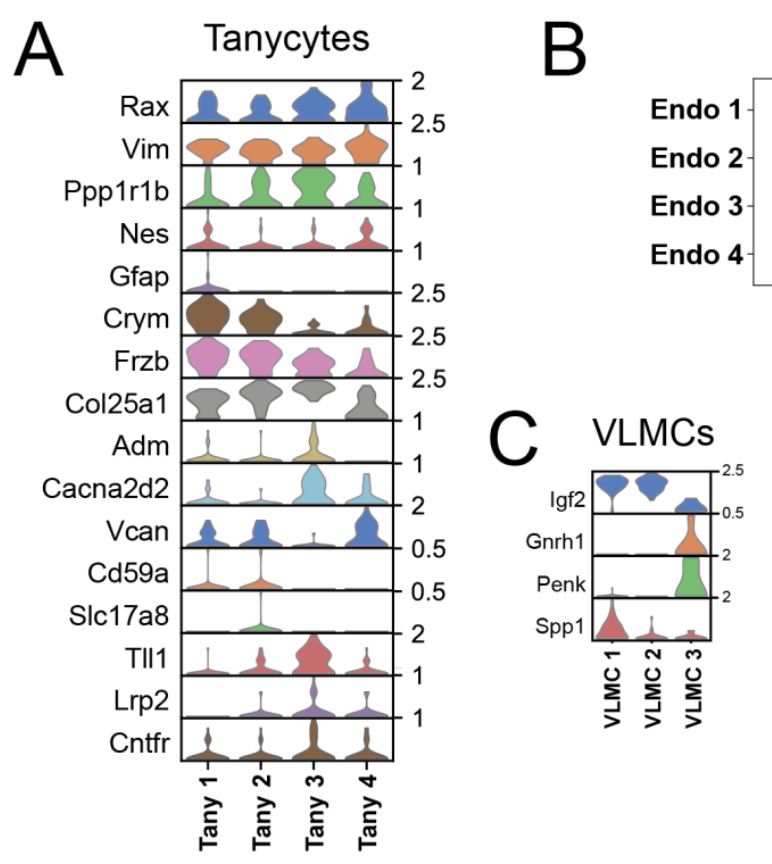

\section{Endothelial cells}
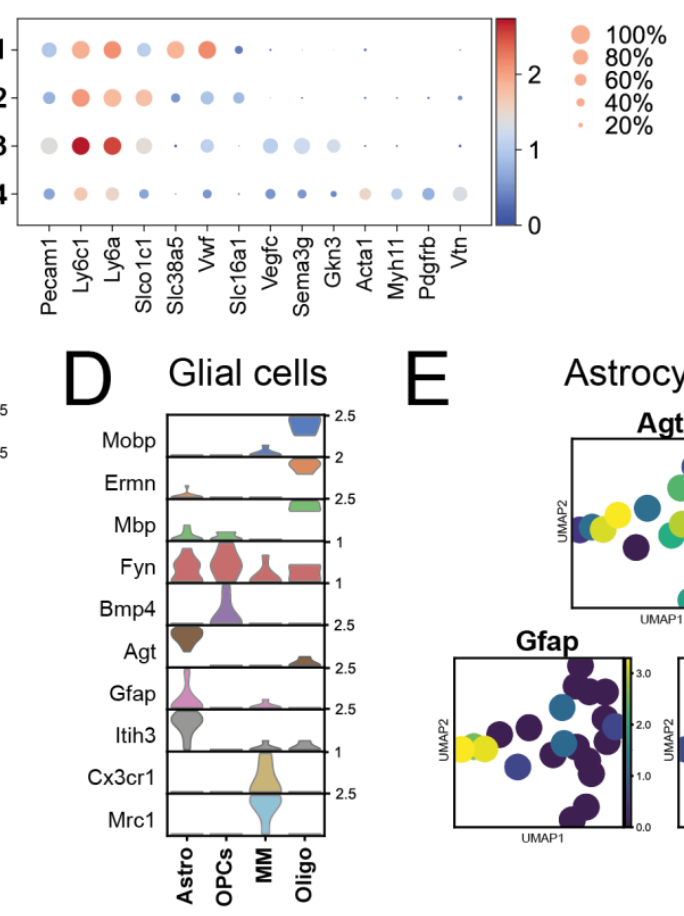

E Astrocytes

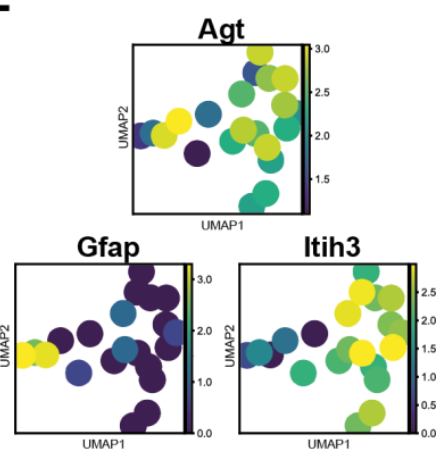




\section{Gene regulatory network of LepR cells}

The scRNAseq atlas of LepR cells in the hypothalamus allows us to investigate the molecular mechanisms of their action. Gene regulatory networks (GRNs) are driven by transcription factors that regulate the expression of sets of target genes (gene modules) in a context-dependent manner. We used the pySCENIC algorithm to determine activity of 'regulons', groups of genes that are co-expressed with a transcription factor that drives their expression, in single cells ${ }^{68,69}$. We detected 38 regulons that display a spectrum of activity over LepR cells, which we visualized using t-SNE (Fig. S5a). Regulon activity recapitulated the distribution of cells types, suggesting that the major determinants of cell types are captured by the GRNs (Fig. 5a).

Next, we performed hierarchical clustering to group cell types using their regulon activity (Fig. 5b). Neurons, astroglia and non-neural cells formed clearly distinct branches in the hierarchy, and subgroups of cell types clustered together (Fig. 5b). Next, we interrogated the regulation networks that were differentially active between different branches of the LepR cell hierarchy using the Mann-U-Whitney test

(Fig. 5b, see Methods). Npdc1 and Smarcc2 regulons discriminated the neuronal cells from astroglia, which was enriched in Ets1 and Erg regulons. Among the neuronal cell types, higher activity of Fli1 and Bclaf1 further defined Agrp neurons. These analyses provide a rich source of regulon activity (Fig. S5b).

\section{Figure 5}
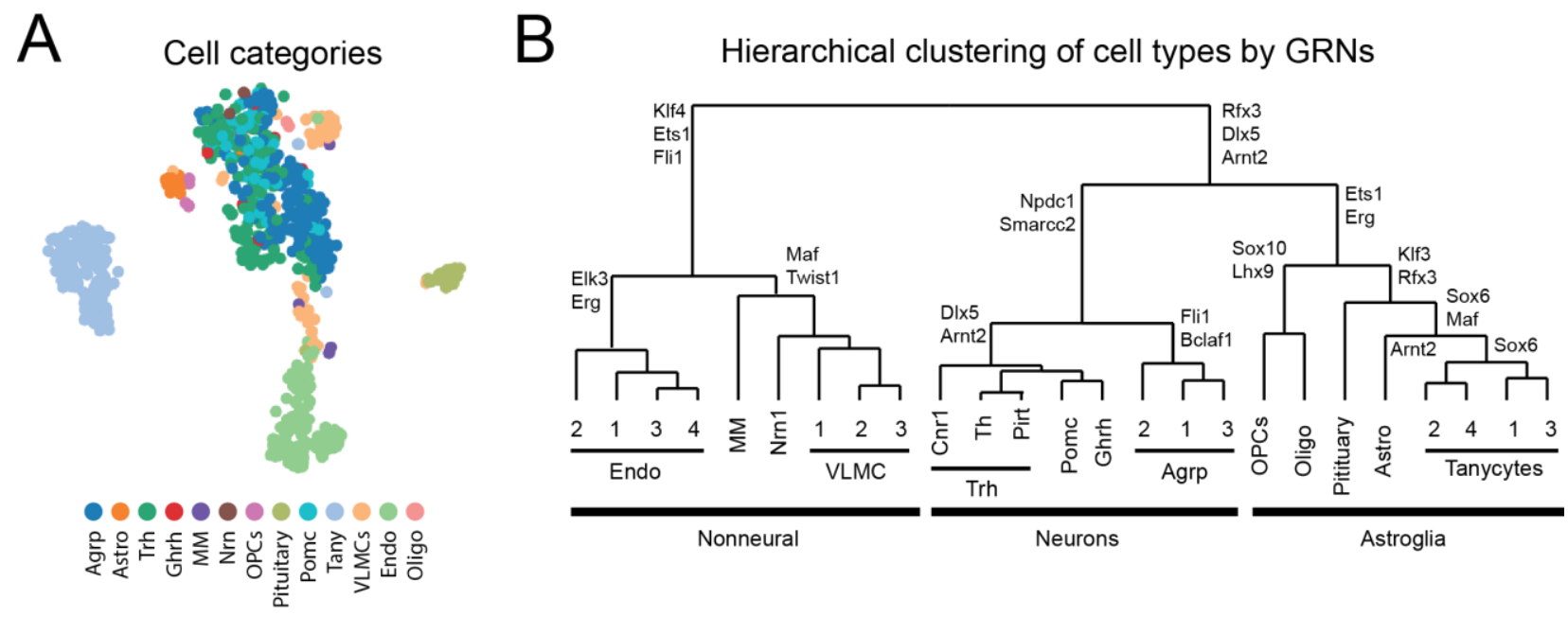


\section{Regulation of LepR-Agrp neurons by fasting}

Next, we investigated the transcriptional response of LepR/tdTomato hypothalamic cells to energy deficit (24-hour fasting). Among the clusters, we saw that Agrp neurons responded the most to fasting, with the highest number of genes being differentially expressed (not shown). We cumulated the Agrp clusters to delineate the effect of fasting on LepR cells. Within the Agrp neuron population fasting induced upregulation of Lepr, Agrp and Npy (Fig. 6a). Using Wilcoxon rank sum test, we identified 93 genes upregulated and 13 genes downregulated by fasting with $\log 2 \mathrm{FC}>1.5$ and $<-1.5$ respectively (sup. Table 2 ). Confirming previously identified genes in Agrp neurons ${ }^{8}$, fasting also induced upregulation of Ghsr, Mt1, Acvr1c and Vgf (Fig. 6a), all of which are involved in obesity and energy balance ${ }^{70-73}$ and downregulation of circadian clock genes Nr1d2, Per3 and Bhlhe40 (Fig. 6b).

Among the upregulated genes (Fig. 6a) we find the transporters SIc4a4 and SIc8a1, the receptors Ghr with a role in hepatic glucose production ${ }^{74}$, Sorcs 1 and $\operatorname{Trpm} 3$ with a role in diabetes, energy balance and temperature sensing ${ }^{75-77}$ as well as the cation channel Trpv2 which regulates BAT thermogenesis ${ }^{78}$. Other upregulated genes that have a relation to obesity and energy balance are Sema3a, Arrb1, Asb4 and Fkbp $5^{79-83}$.

Next, we asked whether we can identify the GRNs involved in response to fasting in Agrp neurons. We reanalyzed the cells from Agrp clusters, which had a median of $\sim 32000$ reads and $~ 9000$ genes per cell, and identified 10 regulons using pySCENIC (Fig. S6a, see Methods). Comparison of fed and fasted Agrp neurons revealed that $\operatorname{Rfx} 3(p=0.000012)$ and $\operatorname{Npdc1}(p=0.00016)$ were the most significantly up- and down- regulated regulons upon fasting, respectively (Fig. 6c). While Rfx3 ( $p=0.0038$ ) mRNA expression was higher in fasted cells, Npdc1 ( $p=0.756)$ mRNA expression was not significantly different between groups, suggesting that the activity of the latter is controlled post-transcriptionally (Fig. S6b).

Regarding the target genes of Rfx3 and Npdc1 (sup. Table 2), we explored their association with energy balance. A target of both Rfx3 and Npdc1, Mapk10 (also known as JNK3), with a role in in energy balance and insulin resistance ${ }^{84-87}$, protects against excessive adipocity ${ }^{88}$. Its selective deficiency from LepR Agrp neurons causes hyperhagia ${ }^{89}$. A downstream target of Rfx3 is the BDNF receptor Ntrk2 (also known as TrkB) which maintains negative energy balance mainly by suppressing feeding ${ }^{90-94}$. Significant hits also were the Npdc1 targets Clstn3 and Faim2. Clstn3 is a key gene that mediates the neuro-adipose junction formation or remodeling in white adipose tissue browning and the BAT activation process ${ }^{95-97}$, while Faim2 is a GWAS gene associated with obesity and its expression is increased in the Arc upon high-fat diet ${ }^{98-100}$. These results further demonstrate the use of our dataset for delineation of the molecular mechanisms governing LepR neuronal activity. 


\section{Figure 6}

A

Genes upregulated by fasting in Agrp neurons

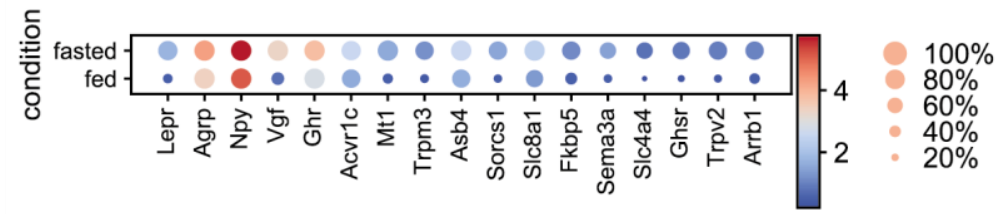

B Genes downregulated by fasting in Agrp neurons

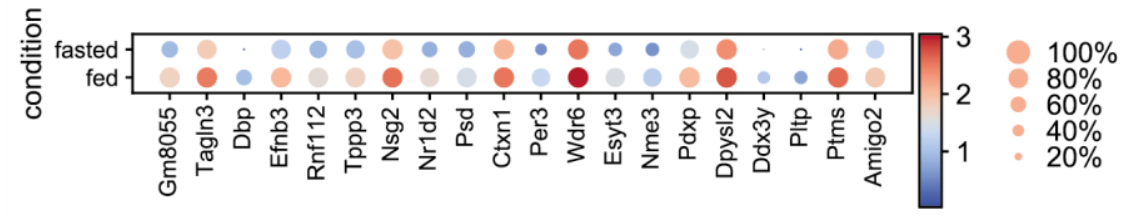

Regulon enrichment fasted vs fed Agrp neurons

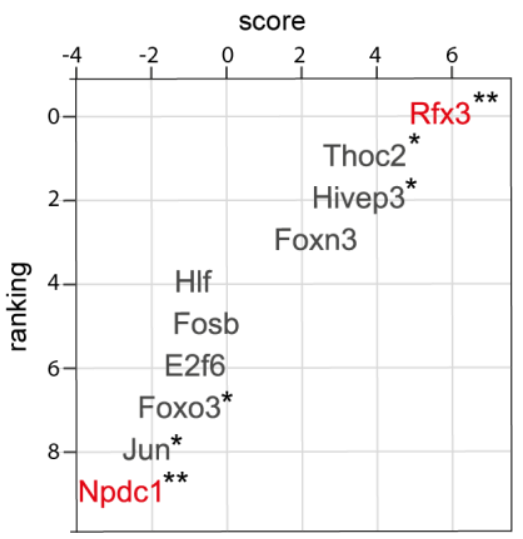




\section{LepR cell enrichment for BMI GWAS genes}

We next asked whether genes specifically expressed within the LepR cell types enriched for genes implicated by genome-wide association study data for obesity (GWAS). Towards that end we first used CELLEX to identify specifically expressed genes for each cell type and then applied CELLECT ${ }^{101}$ to compute the enrichment of GWAS signal for each set of cell type-specific genes. As genetic association data for obesity we relied on UK Biobank-based GWAS summary statistics for body-mass index (BMI), a commonly used proxy phenotype for obesity ${ }^{102}$. CELLECT identified three significantly enriched cell types, namely Trh_Cnr1, Trh_Pirt and Pomc_KNDy neurons (Bonferroni P-value threshold, P<0.05/25; Fig. 7). Together, these analyses confirm the importance of POMC neurons in obesity and reveal the novel LepR clusters Trh_Cnr1 and Trh_Pirt as potential key important players.

\section{Figure 7}

\section{CELLECT on BMI GWAS}

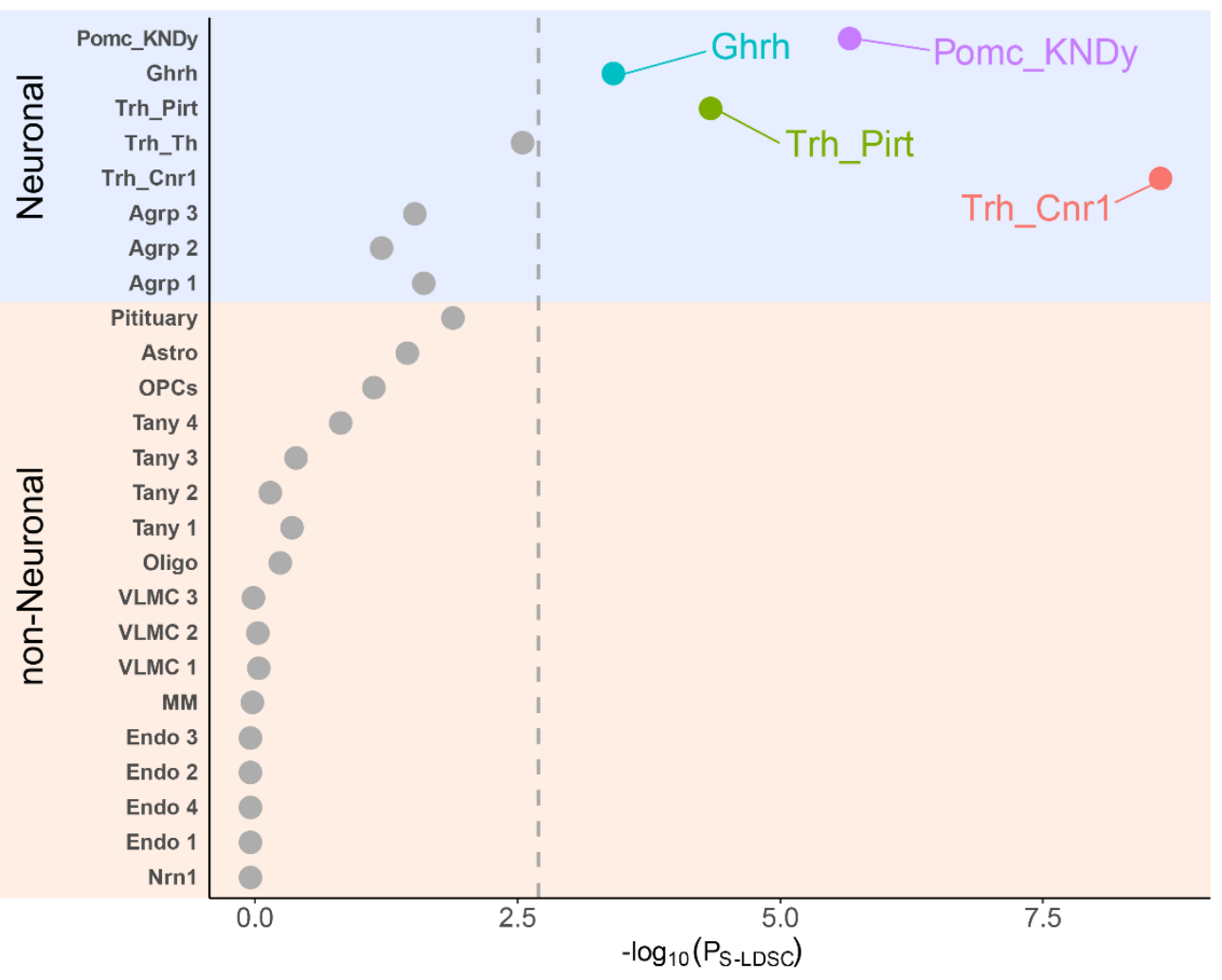




\section{Discussion}

We here identified the transcriptome of hypothalamic LepR cells using scRNAseq. Most available scRNAseq datasets focus on one of the hypothalamic subregions and undertake an unbiased approach to identify all cell populations. Instead, we dissociated the whole hypothalamus of LepR-Cre x L-tdTomato mice and focused solely on the leptin receptor-expressing cells. High sequencing depth combined with FACS sorting of tdTomato-positive cells facilitated the identification of twenty-five distinct cell clusters, including eight neuronal and seventeen non-neuronal.

Overall, our dataset highlights the importance of the arcuate nucleus as the primary action site of leptin, with most of the neuronal LepR-expressing cells in this dataset belonging to at least six distinct neuronal populations localized in the Arc as well as tanycyte subtypes which are in close contact to the Arc and ME. Out of the eight neuronal clusters identified, five (Agrp 1-3, Ghrh and Pomc/KNDy) have already been identified as LepR subpopulations, while three (Trh_Th, Trh_Pirt and Trh_Cnr1) are novel. Even though LepR has been identified in Ghrh neurons before ${ }^{30}$, here we show that all neurons in this cluster also express Th and Gal, revealing a distinct LepR-Arc population with a unique neurochemical profile.

The three newly-identified neuronal clusters displayed Trh expression (Trh_Th, Trh_Cnr1, Trh_Pirt) and they appeared to be quite heterogeneous. The enriched genes in these clusters localize in many hypothalamic nuclei based on Allen Brain Atlas ISH data and literature. The Trh_Th cluster, with high expression of Trh, Th and Cartpt is highly similar to the Arc leptin-sensing GABAergic Trh/Cxcl12 cluster identified by Campbell et al. Therefore, Trh_Th represents another distinct LepR population in the Arc, even though we cannot exclude the fact that Trh_Th neurons could also be localized in other hypothalamic regions (DMH, PVH, PON, LH), due the expression of receptors Ptger3, Adra1b and the chemokine Cxcl12. In the Trh_Cnr1 we identified cells expressing Bdnf, as well as LepR markers of the LH Nts, Gal, Tac1 and Crh. Remarkably, BDNF neurons in the DMH and VMH do not engage into pSTAT3 signaling upon leptin administration, even though Bdnf mRNA is increased ${ }^{103,104}$. While it is known that Gal and Nts are coexpressed in LepR neurons in the $\mathrm{LH}^{53}$, we also found evidence that $\mathrm{Crh}$ also co-localizes with this neuronal cell type. The expression profiles of this cluster's enriched genes support that the majority of the neurons are located in the $\mathrm{VMH}, \mathrm{DMH}$ and $\mathrm{LH}$, skipping the Arc.

The Trh_Pirt cluster displayed high expression of Cartpt, Crhr2 and Pirt, with the two latter genes being expressed mostly in Arc and VMH. CRFR2 in the VMH has been shown to be critical in feeding and regulation of lipid metabolism in mice ${ }^{105}$. Pirt-KO female mice display an increased susceptibility to develop obesity and glucose intolerance ${ }^{54}$. While on average the cells we analyzed were deeply sequenced, it is 
likely that sequencing of a higher number of cells may lead to identification of sub-clusters of the cell types we have identified.

Regarding non-neuronal clusters, we identified LepR populations that are important for leptin transport across the BBB (tanycytes, endothelial cells \& VLMCs) as well as other glial populations. Tanycyte clusters contained mostly $\alpha 2$ - and $\beta 1$-and $\beta 2$ - tanycytes. The presence of leptin receptor in other non-neuronal cell types, highlights that LepR is not only crucial for leptin transport, but also exerts other functions. Leptin signaling in hypothalamic astrocytes is important for synaptic plasticity and neuroendocrine control of feeding by leptin ${ }^{18}$. Moreover, leptin receptor expression on other glial cells (microglia, macrophages, oligodendrocytes and OPCs) suggests that leptin has a neuroprotective role and it is involved in remyelination, as suggested in literature ${ }^{21}$.

With SCENIC analysis we detected 38 active gene regulatory networks which recapitulated the distribution of cell types among clusters and identified regulons that discriminated cell types from each other, such as Fli1 and Bclaf1 which determined Agrp neurons. Agrp neurons was the population that responded the most to fasting, by increasing the expression hormone receptors involved in energy balance and temperature sensing $(G h r, G h s r$, Sorcs1, Trpm3) and decreasing the expression of circadian clock genes (Nr1d2, Per3, Bhlhe40), suggesting that fasting makes these neurons more sensitive to changes in feeding status. SCENIC analysis identified Rfx3 and Npdc1 as the regulons that were mostly up- and downregulated by fasting respectively. Their downstream targets Mapk10, Ntrk2, Clstn3 and Faim2 have high association with energy balance and metabolic diseases. Finally, our BMI GWAS enrichment analysis results study confirm the importance of POMC neurons in regulation of energy balance and furthermore they suggest a role for the novel clusters Trh_Cnr1 and Trh_Pirt in regulating body weight.

Thus, the scRNAseq data we provide reveal the heterogeneity of LepR cells in the hypothalamus, including multiple known as well as novel neuronal, astroglial and non-neural cell types. We show that among these LepR cell types, Agrp neurons are the main responders to fasting. We further demonstrate that our dataset can be used to identify the major gene regulatory networks in homeostasis as well as in response to fasting. Overall, we provide a rich source for future studies that dive into the specific roles LepR cell types as well as the molecular circuitry controlling their function.

\section{Acknowledgements}

Part of this work was funded by an NWO Gravitation grant: BRAINSCAPES: A Roadmap from Neurogenetics to Neurobiology: 024.004.012. 


\section{Methods}

\section{Animals}

Adult 2-3 month-old ObRb-IRES-Cre mice (B6.129(Cg)-Leprtm2(cre)Rck/J) reported as LepR-Crecrossed with Rosa-CAG-LSL-tdTomato-WPRE::DNeo (008320 007914, Jackson laboratories, Bar Harbor, ME, US) reported as L-tdTomatoon a C57BI/6J background were used. Mice were housed socially and kept under a 12:12 hr light-dark cycle with lights off at 19:00. Mice were kept at room temperature $(21 \pm 2 \circ \mathrm{C})$ and 40 $60 \%$ of humidity conditions. They were fed with standard chow (Special Diet Service, Essex, UK) and tap water ad libitum. For the 24 hours of fasting protocol, chow was completely removed 24 hours before being killed. Ad libitum mice were male and female and the mice from the fasting protocol were all female. All experiments were approved by the Ani-mal Ethics Committee of Utrecht University and conducted in agreement with Dutch laws (Wet op de Dierproeven, 1996; revised 2014) and European regulations (Guideline 86/609/EEC; Di-rective 2010/63/EU).

\section{Hypothalamus dissection and single-cell dissociation}

In pilot experiments, we optimized dissociation efficiency to obtain viable cells using various conditions including papain, trypsin and accutase A enzymes as well as the use of trehalose, artificial cerebrospinal fluid and several commercially available media. We found that papain was the most optimal enzyme to dissociate hypothalamic tissue with viable tdTomato ${ }^{+}$cells, and generated an optimized protocol. Mice were killed between 09:00 and 12:00 by manual decapitation after isoflurane anesthesia. Brains were rapidly removed and saved in cold HABG $(50 \mathrm{~mL}$ Hibernate A without calcium (Brainbits, Cologne, Germany) supplemented with $1 \mathrm{~mL} \mathrm{B27}$ and $125 \mu \mathrm{L}$ L-Glutamine). Hypothalami were microdissected and cut into 1-2 mm thick pieces. Tissue from 2-3 hypothalamiwas pooled into 1-1.5 mL of HABG containing papain (20 u/mL, Worthington Biochemical, Lakewood, NJ, US). Tissue was broken into smaller pieces by pipetting 2-3 times with a P1000 pipet followed by a fire-polished glass pipette with a wide opening. Tubes were incubated for $15^{\prime}$ at 37 degrees, whilst being agitated at $160 \mathrm{rpm}$. DNAse I (1,000 Kunitz units/mL Worthington Biochemical, Lakewood, NJ, US) was added and tubes were incubated for $15^{\prime}$ at 37 degrees, whilst being agitated at $160 \mathrm{rpm}$. The samples were chilled on ice the following were added: DNAse I (2,000 Kunitz units $/ \mathrm{mL})$, BSA $(1.25 \mu \mathrm{g} / \mathrm{mL}), \mathrm{FBS}(5 \%)$ and EDTA $(0.5 \mathrm{mM})$. Next, samples were triturated for 2-3 times with a fire-polished glass pipette with medium opening followed by 2-3 times with a fire-polished glass pipette with a small opening. The triturated sample was passed through a 100um cell strainer. The strained suspension was washed once with $10 \mathrm{~mL}$ aCSF(92 mMNaCl, $2.5 \mathrm{mMKCl}, 1.2 \mathrm{mM} \mathrm{NaH} 2 \mathrm{PO}$, 30 mM NaHCO3 , 20 mM HEPES, 25 mM Glucose, 3 mM Sodium Ascorbate, 2 mMThiourea, 3 mM Sodium 
Pyrurate) supplemented with $10 \%$ FBS. Cell suspension was centrifuged at $100 \mathrm{~g}$ for 10 minutes at $4{ }^{\circ} \mathrm{C}$. Cell pellet was resuspended in 200-250 $\mu \mathrm{L}$ of collection solution (aCSF containing $150 \mathrm{u} / \mathrm{mL}$ DNAse I, 10 $\mu \mathrm{M}$ Rock inhibitor and 10\% FBS) and filtered through a $70 \mu \mathrm{m}$ strainer before sorting.

\section{Cell sorting}

Cells were sorted into 384 well-plates using FACS (BD Biosciences Aria II), after gating for forward and side scatter, selecting singlets and finally the tdTomato+ cells with high fluorescence. Approximately 0.8-0.9\% of the total single cell population were defined as tdTomato+ cells (Fig. S1). DAPI was not used for the selection of live (DAPl') cells since pilot experiments showed that tdTomato ${ }^{+}$cells were almost exclusively live, which was confirmed after RNA-seq analysis by assessing the number of reads and other quality measures (see below). When the plates were not completely filled with tdTomato+ cells, cells from mouse hypothalamic of wild-type (L-tdTomato or $\mathrm{C} 57 \mathrm{BI} / 6 \mathrm{~J}$ ) mice were used to fill the plates in order to increase the quantity of captured mRNA which helped with production of high-quality libraries. Plates were span at $2000 \mathrm{rpm}$ for $2 \mathrm{~min}$ and frozen at -80 . In total we processed 7 plates, 4 of which yielded high quality datasets which were used for downstream analysis.

\section{Library generation, sequencing and alignment}

Single-cell RNA sequencing was performed with Cel-seq2 at the Single Cell Discoveries (Utrecht, NL), as described by Hashimshony et al., 2016 and Muraro et al., 2016 25,106. Plates contained preloaded barcoded poly-T primers that specifically amplify the mRNA and introduce a $6 \mathrm{bp}$ unique molecular identifier (UMI), an 8bp cell barcode and a T7 RNA polymerase binding site. In addition, plates were pre-loaded with ERCC spike-ins that allowed quality control of reactions in individual wells. Cells were lysed by heating at $65 \mathrm{C}$ for 5 min followed by rapid chilling on ice for 2 min (twice). mRNA was reversed transcribed using Superscript II (ThermoFisher Scientific) and converted into second stranded DNA by DNA polymerase I (ThermoFisher Scientific). Material from each plate was then pooled, cleaned up using Ampure beads (Beckman Coulter, \#A63881) and amplified by in vitro transcription overnight using the MEGAscript T7 transcription kit (ThermoFisher Scientific, \# AMB1334). Following a second round of clean up, amplified RNA was measured using the Agilent RNA 6000 pico chips (Agilent \# 5067-1513), reverse-transcribed using random hexamer primers that introduce Truseq Small RNA kit RP1 primer binding sites (Illumina) and finally converted into DNA libraries using custom rpi primers (RNA PCR Primer Index) adapted from the Truseq Small RNA kit (Illumina) ${ }^{106}$. Following two rounds of Ampure bead clean up and quality control using the Agilent High Sensitivity DNA Kit (5067-4626), libraries were sent for sequencing at the Utrecht Sequencing Facility (USEQ, Life Sciences faculty, Utrecht University) for paired end sequencing (26bp for read 1 and 50bp for 
read 2) by Nextseq500. 5 plates were combined for a full sequencing run, which yielded $\sim 300 \mathrm{M}$ assigned to the libraries.

Alignment of the Celseq2 reads was performed using the mapandgo. Following merging of the reads generated in multiple lanes and removal of the reads that lack Celseq2 barcodes, the data was trimmed using Trimgalore (version $0.4 .3^{107}$ ) that employs cutadapt ${ }^{108}$ to remove the Illumina library adapters and fastQ $C^{109}$ reads with low quality base-calls at the end of the reads. Alignment of the reads to the mm 10 reference genome was done STAR (version 2.5.3 ${ }^{110}$ ), and single-cell libraries were de-multiplexed using the barcoded present in preloaded primer sequences. Following UMI correction, reads were adjusted for the possibility of false assignment of reads from different mRNAs as duplicates due to the low chance of having the same UMI on different mRNA molecules. This was the final count file used for the analysis.

\section{Computational analysis with Scanpy}

We used the Scanpy ${ }^{26}$ pipeline to perform the analysis of our single cell data. We removed cells 2000 genes and with more than $50 \%$ of the reads belonging to ERCC spike-in RNA (indicative of poor caption of mRNA from cells) or with more than 15\% mitochondrial gene expression (indicative of RNA degradation and poor cell viability) which left 824 cells (out of 1288) for analysis, 692 (out of 1048) cells from the fed condition and 132 cells (out of 240 ) from the fasted condition. Genes expressed in only one single cell, the Malat1 gene (due to mapping issues), the ERCC spike-ins and the mitochondrial RNAs were removed from the dataset. This resulted in a high-quality dataset containing a median of 12082 unique counts and 4756 genes per cell.

\section{Selection of normalization method}

Following $\log (1+p)$ transformation and log normalization of the data, we observed that a fourth separate $\mathrm{Agrp}^{+}$cluster was composed exclusively of cells from the fasted condition with higher levels of total read counts. The fraction of ERCC spike-in reads were similar ( 9\%) between clusters, suggesting that the difference between fed and fasted conditions is not due to a higher amount of total RNA in fasted cells, but due to deeper sequencing (see respective Github page). To test this, we downsampled the reads from each cell to 3000 reads prior to the analysis, which removed the technical differences due to sequencing depth and resulted in fed and fasted cells clustering consistently among the three Agrp clusters. From here on, we used the downsampled dataset and considered the differences as biological. For the analysis of differences between fed and fasted condition in Agrp neurons, we reanalyzed the raw data by downsampling to the median ( 39,000 reads per cell). We regressed out the effect of number of genes and the mitochondrial gene expression from both datasets. The data was log-transformed and scaled prior 
to PCA analysis, which was used for dimensionality reduction using t-distributed stochastic neighbor embedding (t-SNE). Visualization of cell clusters and gene expression was performed using UMAP dimensionality reduction plots, the coordinates of which was used to calculate cell clusters using the Leiden algorithm ${ }^{111}$. Differential gene expression between clusters was performed using the integrated rank_gene_groups() function and the Wilcoxon rank-sum test. Using these marker genes, we annotated cell types. To compare the annotated cell types to the literature, we used the online platform Scibet ${ }^{67}$. The details of clustering, calculation of differential gene expression as well as the genes used for the figures in this paper can be found on https://github.com/neuronur/scLepr.

\section{Marker gene identification}

Spatial expression of the identified genes was investigated from publicly available ISH from the Allen Brain Atlas. Furthermore, for the identified genes PubMed search was applied for literature related to food intake, energy, leptin (receptors) and the hypothalamus. Lowly expressed genes are notoriously hard to detect in scRNAseq, which result in dropouts (zero values in many cells). When expression of rare genes within a cluster is normally distributed, we can consider that the gene is expressed by all cells within the cluster, which our methods stochastically (or randomly) detect.

\section{Cell type annotation using SciBet}

We used the online classification tool (http://scibet.cancer-pku.cn/download references.html) of SciBet, which employs the E-test to predict similar cell types between the query and a reference. For this, we only included the highly variable genes from the Lepr scRNAseq dataset and used manually annotated cell type names for each cluster determined by the Leiden algorithm. For comparison to Chen et al., the reference dataset provided by the tool was used. For comparison to Campbell et al., we reanalyzed the dataset and exported a data frame of 2000 top highly variable genes and 2000 randomly selected cells. Cell type annotation was provided with the dataset. Details of the preprocessing of the data frames for the analysis can be found at https://github.com/neuronur/scLepr.

\section{Identification of gene regulatory networks}

We used the pySCENIC 68,69 pipeline to identify gene regulatory networks. In brief, the raw data from all tdTomato ${ }^{+}$cells or Agrp neurons were used to calculate regulons that contain transcription factors and their targets that are expressed in the same cell using the arboreto package ${ }^{112}$. These regulons were then pruned for targets that lack a binding motif for the respective transcription factor within the $5 \mathrm{~kb}$ of the transcription start site. The activity of these regulons for each cell were integrated in an Anndata object containing the single cell expression data generated by the scanpy pipeline, as described above. When 
clustered based on regulon activity, cell types clustered similarly to the analysis based on whole transcriptome. To find out which regulons were driving the branching of cell types upon hierarchical clustering, we used the Wilcoxon rank-sum test. The differences between fed and fasted conditions in Agrp neurons were calculated similarly. A detailed description of the analysis and the code used is available on https://github.com/neuronur/scLepr.

\section{CELLECT analysis}

We used CELLECT v.1.1 ${ }^{113}$ to identify likely etiologic cell-types underlying obesity. CELLECT takes as input GWAS data and cell-type expression specificity (ES) estimates. The output is a list of prioritized etiologic cell-types for a given complex trait. To generate the ES estimates we used CELLEX v.1.1 ${ }^{114}$. CELLEX computes robust estimates of ES relying on multiple expression specificity measures (for details see ${ }^{101}$ ). CELLEX was run using the raw gene expression matrix and the metadata containing clustering information. The resulting cell-type specificity matrix along with the UKBB BMI GWAS ${ }^{102}$ was used as input for CELLECT which was run with default parameters. For the associations of our dataset to additional GWAS studies, the following resources were used: ${ }^{115-123}$ \& Alkes group at the Broad institute and https://www.med.unc.edu/pgc/download-results/ed/. Significant cell-types were identified using a Bonferroni $p$-value threshold of $p<0.05$.

\section{Cloning and DIG probe labeling}

PCR with forward and reverse primers (see Table 1) was performed on mouse hypothalamic cDNA (isolated with miRNeasy Mini Kit, Qiagen, Hilden, Germany). PCR products were ligated into pGEMT.easy (Promega, Madison, WI, US) and sequenced with Sanger sequencing. PCR with SP6 and T7 primers was performed. cDNA probes were incubated for 2 hours at 37 degrees with Dig RNA labeling mix (11277073910, Roche, Basel, Switzerland) and SP6 or T7 RNA polymerase (RPOLSP6-RO and RPOLT7-RO, Roche).

$\begin{array}{lll}\text { Gene } \quad \text { Fw sequence } & \text { Rv sequence } & \text { Ann. temp }\end{array}$

\begin{tabular}{llll}
\hline Gal & AtCCAGCCCGCCACTCtTCA & ACAGCtTCAAAGCAGAGAACAGA & $59^{\circ} \mathrm{C}$ \\
Ghrh & CCTTCAGGATGCAGCGACAC & CGgAAAAGGTCAGAGCTGAAG & $47^{\circ} \mathrm{C}$
\end{tabular}

Table 1: Primer sequences and annealing temperatures for PCR

\section{Brain collection and sectioning for histology}


LepR-Cre $x$ L-tdTomato mice were perfused with PBS $1 x$ followed by $4 \%$ paraformaldehyde (PFA) in PBS 1x. Brains were sliced coronally at $20 \mu \mathrm{m}$ thickness in a cryostat (Leica Biosystems, Wetzlar, Germany) with chamber temperature of $21 \pm 3^{\circ} \mathrm{C}$ and object temperature of $19 \pm 3^{\circ} \mathrm{C}$ and sections were mounted directly on Superfrost glass (631-0108, VWR, Leuven, The Netherlands) in series of 10 per each brain and stored at $-80^{\circ} \mathrm{C}$.

\section{Fluorescent in situ hybridization and immunofluorescence for tdTomato}

Slides were thawed at room temperature (RT) for 1 hour. Sections were incubated with 1,32\% triethanolamine and $0,18 \% \mathrm{HCl}$ for 10 at $\mathrm{RT}$ and with hybridization mix ( $50 \%$ deionized formamide, $5 \times$ SSC buffer, $5 x$ Denharts, $250 \mu \mathrm{g} / \mathrm{ml}$ tRNA baker's yeast and $500 \mu \mathrm{g} / \mathrm{ml}$ Sonificated Salmon Sperm DNA) for 2 hours at RT. Sections were incubated with RNA probes (400 ng/mL hybridization mix) overnight at 68 degrees. Slides were transferred to $2 \times$ SSC at 68 degrees and then immediately to $0.2 \times$ SSC at 68 degrees for 2 hours. After that, sections were treated with 0,3\% hydrogen peroxide in 1x TBS for 30' at RT, blocked with TNB blocking buffer (from TSA Plus Cyanine 3 System, NEL744001KT, PerkinElmer, Waltham, MA, US) for $1 \mathrm{hr}$ at RT and incubated with anti-DIG-POD (1:500, 11207733910, Roche) and rabbit anti-RFP (1:1000, 600-401-379, Rockland, Limerick, PA, US) in TNB blocking buffer overnight at 4 degrees. Sections were then incubated with Cyanine 3 Tyramide amplification reagent (1:50 in 1X amplification diluent) from TSA kit for 10-15' at RT followed by incubation with goat anti-rabbit 568 (1:500, ab175471, Abcam, Cambridge, UK) in TNB blocking buffer and DAPI (1:1000 in 1x PBS). Between steps sections were washed 4x5' with 1xTNT buffer (0,1M Tris- $\mathrm{HCl}$, pH7.5, 0,15M NaCl, 0,05\% Tween20). Sections were let to dry and covered with Fluorsave reagent (Calbiochem, San Diego, CA, US).

\section{Immunofluorescence}

Slides were thawed at room temperature (RT) for 1 hour. Slides were incubated with blocking solution (5\% normal goat serum (NGS), $0.5 \%$ Triton X-100 in $1 \mathrm{x}$ PBS) for 1 hour at room temperature (RT), followed by 2 hours incubation at RTwithRabbit anti-TH (Milipore, Ab152)1:500 diluted in carrier solution (5\% NGS, 0,1\% Triton X-100 in 1x PBS). Sections were then incubated with Alexa fluor 488 Goat a-rabbit (ab150077, Abcam) 1:1000 diluted in carrier solution for 2 hours at RT. Between all steps sections were washed 3 times for 5-10 minutes in PBS 1x. Sections were let to dry and covered with Fluorsave (Calbiochem, San Diego, CA, US).

\section{Imaging and Image analysis}


10x magnification pictures were taken with an epi-fluorescent microscope (Zeiss Scope A1, ZEISS, Germany). Hypothalamic structures were defined based on the The Mouse Brain in Stereotaxic Coordinates, 3rd Edition ${ }^{124}$.

\section{Data and code sharing}

The scRNAseq raw data is uploaded to GEO (\#XXX) in the format of fastq files and a preprocessed dataset as a csv file. Data of Campbell et al was downloaded from GEO (GSE93374). All the code used for the analysis in the single cell data as well as the codes to reproduce the figures of the manuscript is deposited to Github (https://github.com/neuronur/scLepr). 


\section{Figure S1}

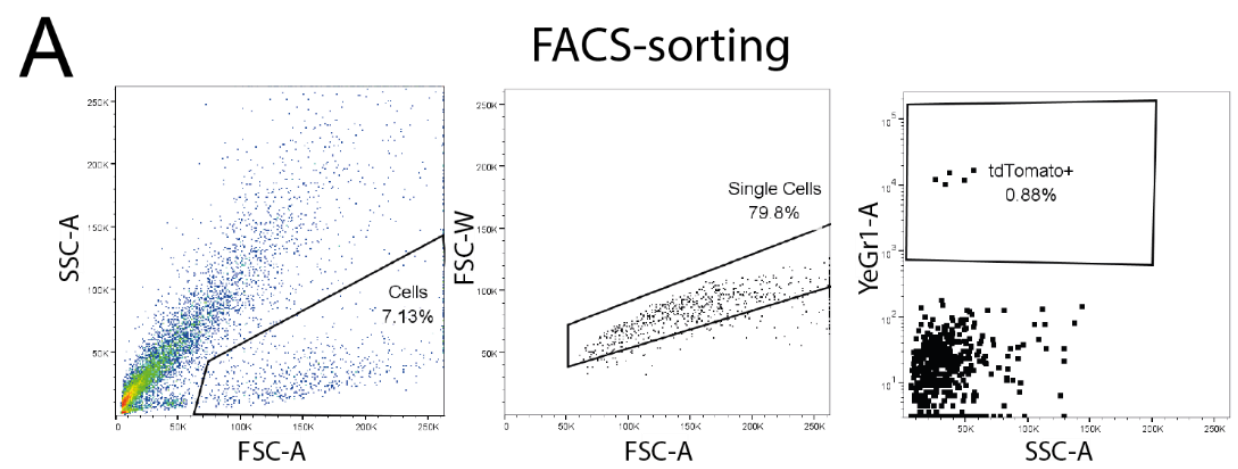

B

Batches and conditions used
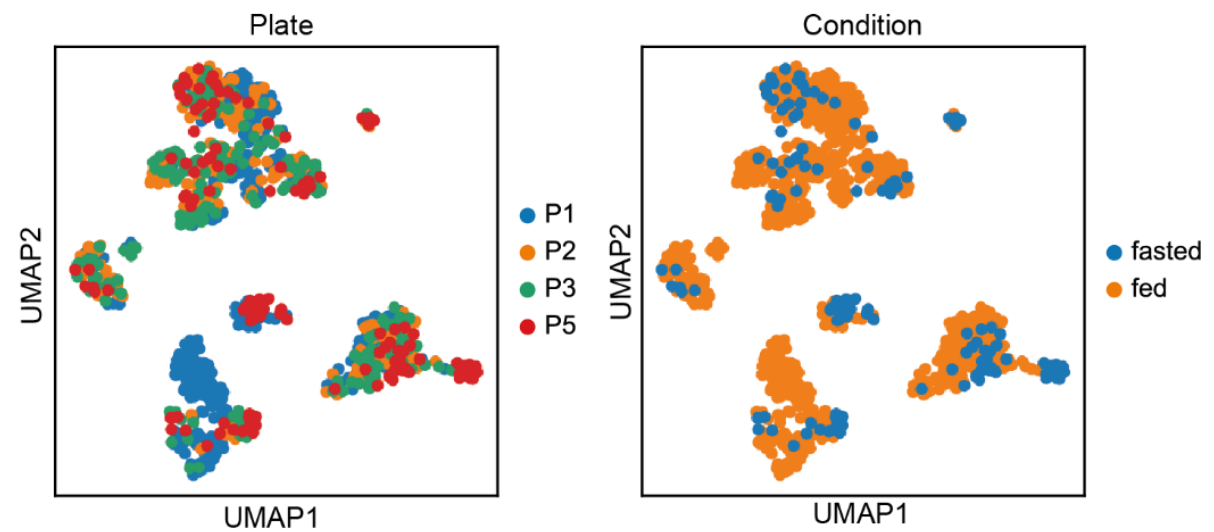

C

\section{Quality control measures}
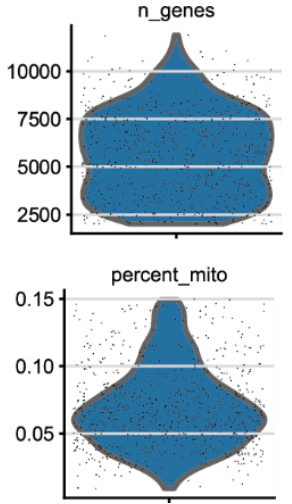
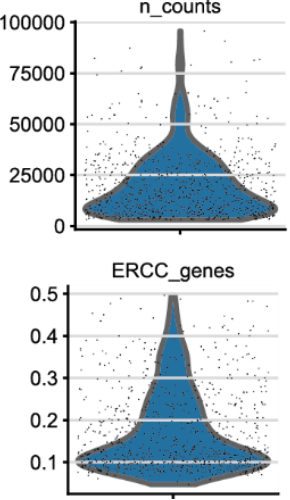

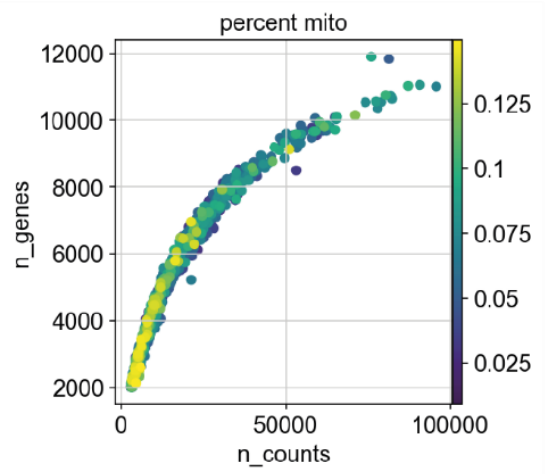




\section{Figure S2}

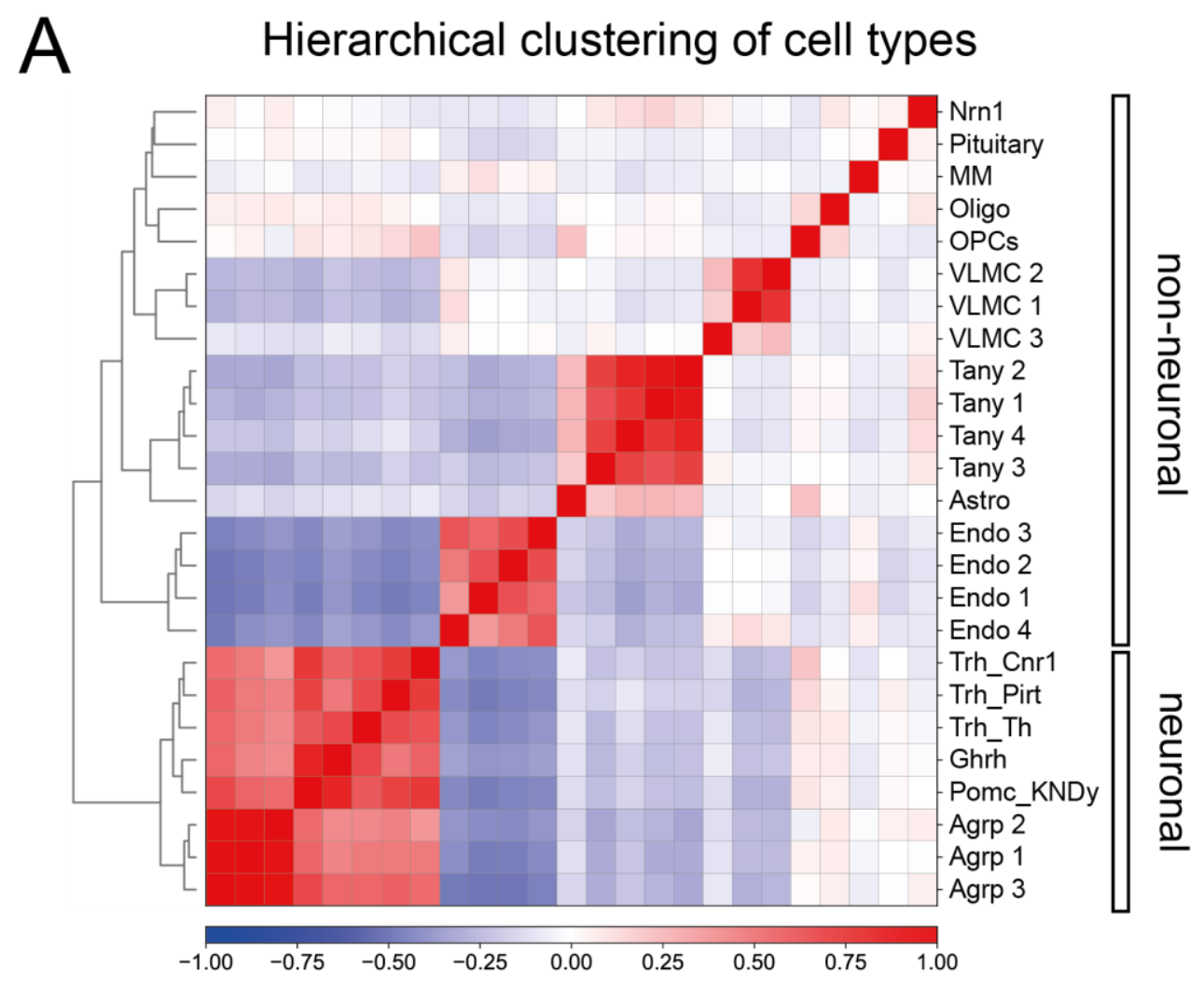

B

\section{Lepr expression}

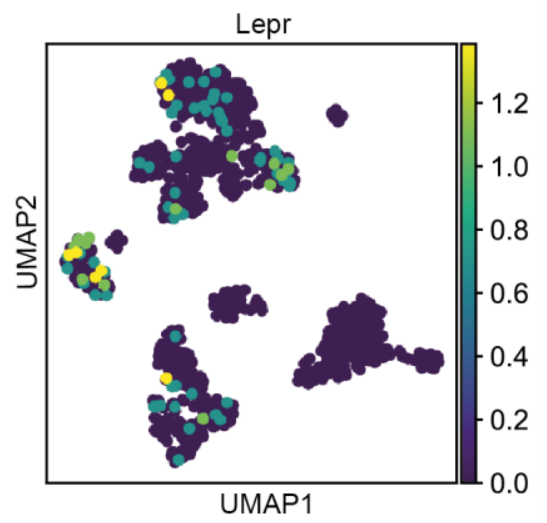


bioRxiv preprint doi: https://doi.org/10.1101/2020.07.23.217729; this version posted July 24, 2020. The copyright holder for this preprint (which was not certified by peer review) is the author/funder, who has granted bioRxiv a license to display the preprint in perpetuity. It is made available under aCC-BY-NC-ND 4.0 International license.

\section{Figure S3}

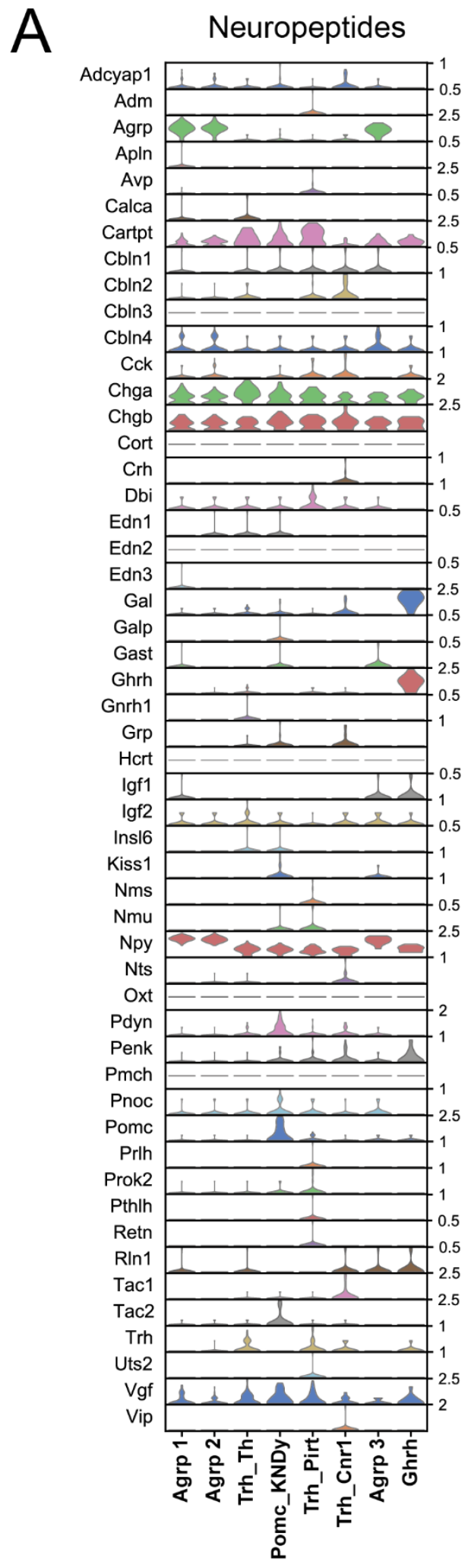

\section{B}

Neuropeptide receptors

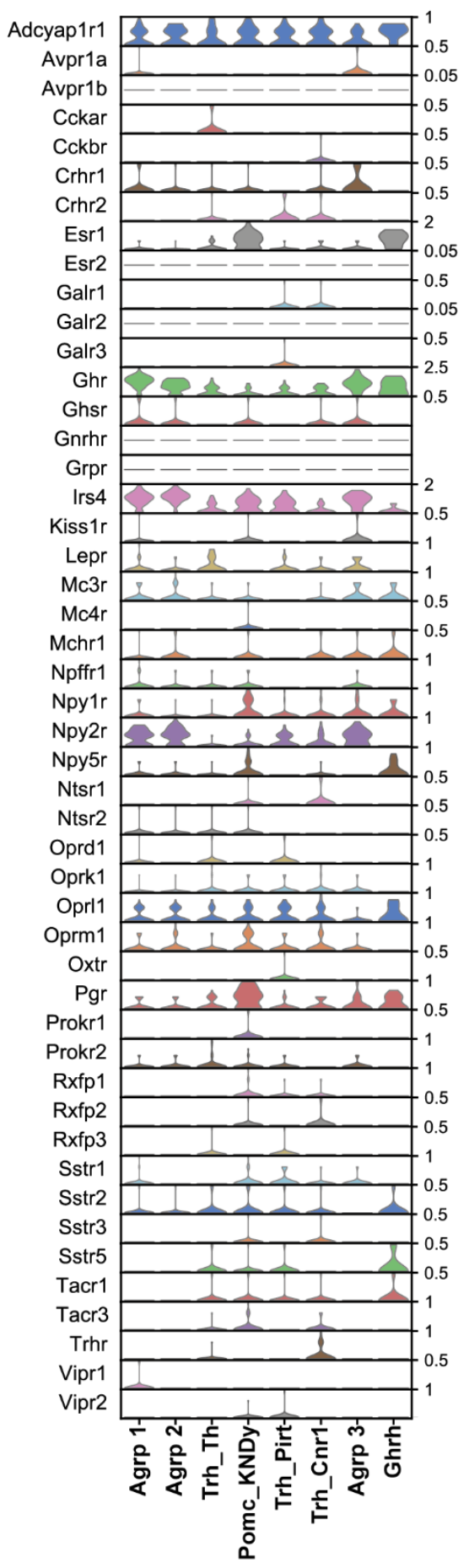


bioRxiv preprint doi: https://doi.org/10.1101/2020.07.23.217729; this version posted July 24, 2020. The copyright holder for this preprint (which was not certified by peer review) is the author/funder, who has granted bioRxiv a license to display the preprint in perpetuity. It is made available under aCC-BY-NC-ND 4.0 International license.

\section{Figure S4}

\section{Comparison of cell types to \\ Chen et al}

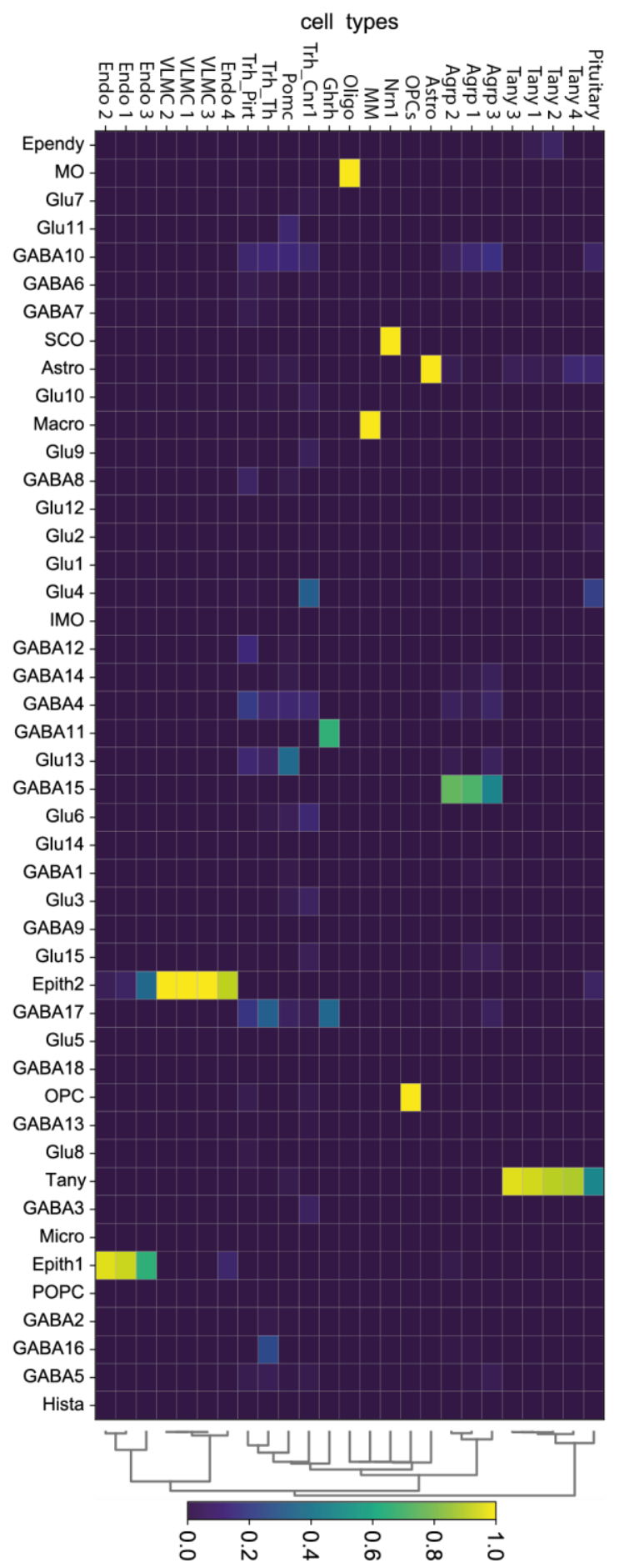


bioRxiv preprint doi: https://doi.org/10.1101/2020.07.23.217729; this version posted July 24,2020 . The copyright holder for this preprint (which

was not certified by peer review) is the author/funder, who has granted bioRxiv a license to display the preprint in perpetuity. It is made available under aCC-BY-NC-ND 4.0 International license.

\section{Figure S6}
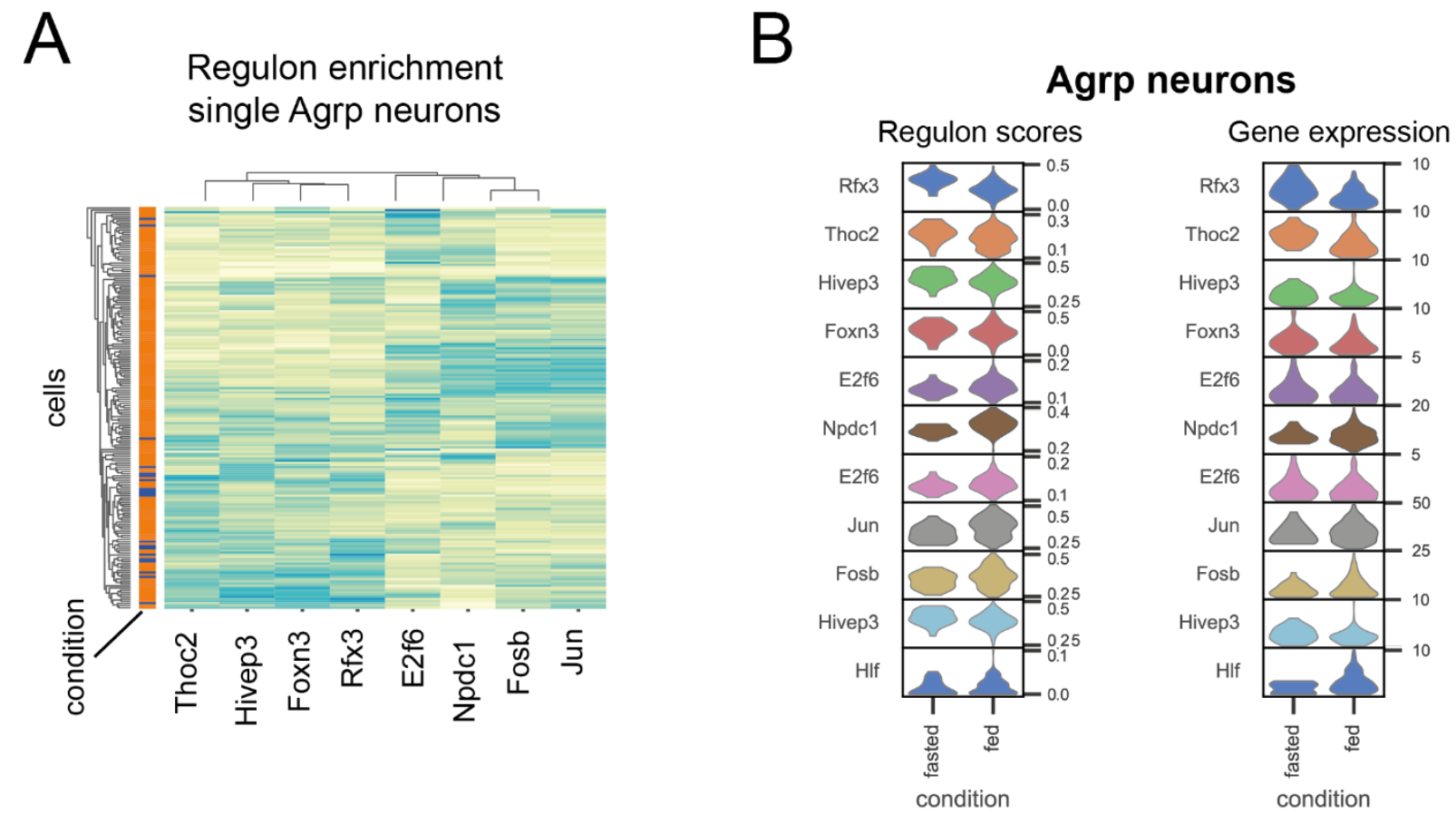


\section{Figure Legends}

Figure 1 scRNAseq on hypothalamic LepR cells reveals 25 clusters.

A. Hypothalami of fed and 24-hour fasted LepR-Cre $\times$ L-tdTomato mice were dissected, dissociated with papain and FACS-sorted into 384-well plates, where the Cel-Seq2 protocol was performed.

B. 25 clusters of hypothalamic LepR cells annotated on a UMAP after clustering analysis.

C. Violin plots of pan marker genes used to define which main cell types the clusters represent. The maximum normalized count of each pan marker gene is presented on the right. Endo: Endothelial, Astro: Astrocytes, Tany: Tanycytes, VLMC: Vascular leptomeningeal cells, MM: Microglia and Macrophages, Oligo: Oligodendrocytes, OPCs: Oligodendrocyte progenitor cells.

Figure 2 Overview of the eight neuronal LepR clusters in the hypothalamus.

A. 8 neuronal clusters of LepR cells annotated on a UMAP plot.

B. Violin plots of expression of genes used to characterize the neuronal clusters, including GABAergic marker S/c32a1, glutamatergic marker S/c17a6, various neuropeptides and receptors. The maximum normalized count of each gene is presented on the right.

C-F. UMAP plots showing expression level of genes in neuronal clusters. The expression level is colorcoded.

Figure 3 Overview of the 3 novel Trh clusters.

A. Left: Dot-plot of genes expressed in the three Trh clusters. Color-coding represents expression levels, dot size represents the percentage of cells expressing this gene. Right: Trh neurons were reanalyzed. The three clusters are shown on a UMAP plot.

B-C. UMAP plots showing expression level of selected genes in Trh clusters. The expression level is colorcoded.

D. Pictures of fixed hypothalamic sections from LepR-Cre $x$ L-tdTomato mice stained with an antibody against tdTomato (green) and fluorescent in situ hybridization (FISH) with anti-sense probe against Ghrh (red).

E. Pictures of fixed hypothalamic sections from LepR-Cre $x$ L-tdTomato mice stained with an antibody against tdTomato (green) and fluorescent in situ hybridization (FISH) with anti-sense probe against Gal (red) 
F. Pictures of fixed hypothalamic sections from LepR-Cre $x$ L-tdTomato mice stained with an antibody against TH (green). tdTomato fluorescence appears in red, there was no staining performed against tdTomato.

White arrows indicate cells in which co-localization is observed. Scale bars: 100 um. Arc: Arcuate nucleus, VMH: Ventromedial hypothalamus, DMH: Dorsomedial hypothalamus, LH: Lateral hypothalamus

Figure 4 Overview of the non-neuronal clusters of LepR cells in the hypothalamus.

A. Violin plots showing the expression levels of selected genes in the four tanycyte clusters.

B. Dot-plot of expression of selected genes in the endothelial clusters. Color-coding represents expression levels and dot size represents the percentage of cells expressing this marker.

C. Violin plots showing the expression levels of genes in the three VLMC clusters. VLMC: Vascular leptomeningeal cells.

D. Violin plots showing the expression levels of genes in Glial cell clusters. Astro: Astrocytes, MM: Microglia and Macrophages, Oligo: Oligodendrocytes, OPCs: Oligodendrocyte progenitor cells.

E. UMAP plots showing expression of astrocytic markers in astrocytes. The expression level is color-coded.

In $A, C$ and $D$, the maximum normalized count of each gene is presented on the right.

Figure 5 Gene regulatory network (GRN) analysis of LepR cells in the hypothalamus by pySCENIC.

A. tSNE plot showing cell categories following clustering based on GRN analysis.

B. Dendrogram representing hierarchical clustering based on regulons identified as active by pySCENIC analysis. The main regulons that are driving discrimination of branches (cell types) are annotated. Endo: Endothelial, Astro: Astrocytes, Tany: Tanycytes, VLMC: Vascular leptomeningeal cells, MM: Microglia and Macrophages, Oligo: Oligodendrocytes, OPCs: Oligodendrocyte progenitor cells.

Figure 6 Effect of 24-hour food deprivation on LepR Agrp neurons.

A-B. Dot-plots of a selection of genes identified to be upregulated and downregulated by fasting in LepR Agrp neurons. Color-coding represents expression levels, dot size represents the percentage of cells expressing this marker. In A, selected genes are shown. Besides Lepr, Agrp and Npy displayed on the left, the rest of the selected genes are ordered from left to right by lowest to highest $p$ value. In $B$, the top 20 downregulated genes are ordered from left to right by lowest to highest $p$ value.

C Scatter plot showing how regulon activity is affected by fasting in LepR Agrp neurons, ranked by regulon score. p-adjusted: $* *<0.001, *<0.05$, following Mann-Whitney-Wilcoxon test. 
Figure 7 Scatterplot of the CELLECT results. Dashed vertical line marks the Bonferroni threshold for significant $p$-values. The $y$-axis shows both clustering names and overall cell-type, and the $x$-axis shows the p-values. Only cell-types that are significant are shown in color Bonferroni P-value threshold $(P<0.05 / 25)$. S-LDSC, stratified-linkage disequilibrium score regression. Endo: Endothelial, Astro: Astrocytes, Tany: Tanycytes, VLMC: Vascular leptomeningeal cells, MM: Microglia and Macrophages, Oligo: Oligodendrocytes, OPCs: Oligodendrocyte progenitor cells.

Figure S1 FACS-sorting, plate/batch and condition distribution and quality measures

A Plots representing the selection of cells to be sorted in 384-well plates, example from a single run. Left: $7,13 \%$ of the population was selected from the forward (FSC) and side scatter (SSC). Middle: $79,8 \%$ of cells were selected as singlets. Right: $0.88 \%$ of singlets were selected as tdTomato-positive based on fluorescence detected by the yellow laser.

B UMAP plots showing distribution of plates (batches) and conditions

C Plots representing quality measures for the number of genes, counts, percentage of mitochondrial genes and fraction of ERCC spike-in genes. The plot on the right show the correlation between the number of genes and counts as a measure of complexity. Cells are colored with the percentage of mitochondrial genes detected.

Figure S2 Hierarchical clustering of clusters and Lepr expression

A Heatmap showing Euclidean distances between clusters. The dendrogram depicts hierarchical clustering.

B UMAP plot of Lepr gene expression. The expression level (normalized counts) is color-coded.

Figure S3 Neuropeptide and neuropeptide receptor expression in neuronal clusters of LepR neurons in the hypothalamus.

A-B Violin plots of expression of neuropeptide (A) and neuropeptide receptor genes (B) in neuronal clusters. The maximum normalized count of each gene is presented on the right.

Figure S4 Comparison of Lepr cell types to the literature

Matrixplot showing the predicted probability of assignment of Lepr cell types identified in our study to published datasets using the SciBet pipeline. Chen et al identified cell types from the whole hypothalamus. Endo: Endothelial, Astro: Astrocytes, Tany: Tanycytes, VLMC: Vascular leptomeningeal cells, MM: Microglia and Macrophages, Oligo: Oligodendrocytes, OPCs: Oligodendrocyte progenitor cells.

Figure S5 pySCENIC analysis for identification of GRNs 
A tSNE plot representing distribution of conditions

B Dot-plot representing the 38 regulons identified to be active in the 25 clusters of LepR hypothalamic cells. Color-coding represents average regulon scores per cell and dot size the percentage of cells in which the regulon is active.

Figure S6 GRNs regulated by fasting in LepR Agrp neurons

A Heatmap representing regulon activity in individual LepR Agrp neurons from the fed and fasted condition. Color-coding represents regulon score.

B Violin plots of regulon scores (numbers represent min and max) and gene expression levels (numbers represent max) in the fed and fasted condition

\section{Table legends}

Sup. Table 1 Differential expression analysis of genes in Agrp LepR cells of the fed and fasted condition. Mean: mean counts in fed/fasted, pct: fraction of cells expressing the gene, s: score, I: log2FoldChange, $\mathrm{p}$ : adjusted $\mathrm{p}$ value.

Sup. Table 2 Target genes of each regulon identified to be differentially regulated upon fasting in Agrp LepR cells. 


\section{References}

1. Zhang, Y. et al. Positional cloning of the mouse obese gene and its human homologue. Nature 372, 425-432 (1994).

2. Zhang, Y. \& Chua, S. Leptin Function and Regulation. Compr. Physiol. 8, 351-369 (2017).

3. RV, C. et al. Serum Immunoreactive-Leptin Concentrations in Normal-Weight and Obese Humans. N. Engl. J. Med. 334, (1996).

4. Kolaczynski, J. W. et al. Acute and Chronic Effect of Insulin on Leptin Production in Humans: Studies In Vivo and In Vitro. Diabetes 45, 699-701 (1996).

5. MA, C. et al. Leptin Activates Anorexigenic POMC Neurons Through a Neural Network in the Arcuate Nucleus. Nature 411, (2001).

6. Elias, C. F. et al. Leptin differentially regulates NPY and POMC neurons projecting to the lateral hypothalamic area. Neuron 23, 775-86 (1999).

7. $M$, van den T., K, L., AD, W., AM, B. \& D, S. Orexigen-sensitive NPY/AgRP Pacemaker Neurons in the Hypothalamic Arcuate Nucleus. Nat. Neurosci. 7, (2004).

8. Henry, F. E. et al. Cell type-specific transcriptomics of hypothalamic energy-sensing neuron responses to weight-loss. Elife 4, 11929-11947 (2015).

9. Chen, R., Wu, X., Jiang, L. \& Zhang, Y. Single-Cell RNA-Seq Reveals Hypothalamic Cell Diversity. Cell Rep. 18, 3227-3241 (2017).

10. Campbell, J. N. et al. A Molecular Census of Arcuate Hypothalamus and Median Eminence Cell Types. Nat. Neurosci. 20, 484 (2017).

11. Scott, M. M. et al. Leptin targets in the mouse brain. J. Comp. Neurol. 514, 518-32 (2009).

12. Elmquist, J. K., Bjorbok, C., Ahima, R. S., Flier, J. S. \& Saper, C. B. Distributions of leptin receptor mRNA isoforms in the rat brain. J. Comp. Neurol. 395, 535-547 (1998).

13. Nillni, E. A. et al. Leptin regulates prothyrotropin-releasing hormone biosynthesis. Evidence for direct and indirect pathways. J. Biol. Chem. 275, 36124-33 (2000).

14. Dhillon, H. et al. Leptin directly activates SF1 neurons in the $\mathrm{VMH}$, and this action by leptin is required for normal body-weight homeostasis. Neuron 49, 191-203 (2006).

15. Dodd, G. T. et al. The thermogenic effect of leptin is dependent on a distinct population of 
prolactin-releasing peptide neurons in the dorsomedial hypothalamus. Cell Metab. 20, 639-49 (2014).

16. Ghamari-Langroudi, M., Srisai, D. \& Cone, R. D. Multinodal regulation of the arcuate/paraventricular nucleus circuit by leptin. Proc. Natl. Acad. Sci. U. S. A. 108, 355-60 (2011).

17. Hsuchou, H. et al. Obesity induces functional astrocytic leptin receptors in hypothalamus. Brain 132, 889-902 (2009).

18. Kim, J. G. et al. Leptin signaling in astrocytes regulates hypothalamic neuronal circuits and feeding. Nat. Neurosci. 17, 908-10 (2014).

19. Balland, E. et al. Hypothalamic tanycytes are an ERK-gated conduit for leptin into the brain. Cell Metab. 19, 293-301 (2014).

20. Hubert, A. et al. Selective Deletion of Leptin Signaling in Endothelial Cells Enhances Neointima Formation and Phenocopies the Vascular Effects of Diet-Induced Obesity in Mice. Arterioscler. Thromb. Vasc. Biol. 37, 1683-1697 (2017).

21. Fujita, Y. \& Yamashita, T. The Effects of Leptin on Glial Cells in Neurological Diseases. Front. Neurosci. 13, 828 (2019).

22. Matoba, K., Muramatsu, R. \& Yamashita, T. Leptin sustains spontaneous remyelination in the adult central nervous system. Sci. Rep. 7, 40397 (2017).

23. Yuan, X., Caron, A., Wu, H. \& Gautron, L. Leptin Receptor Expression in Mouse Intracranial Perivascular Cells. Front. Neuroanat. 12, 4 (2018).

24. Mickelsen, L. E. et al. Single-cell transcriptomic analysis of the lateral hypothalamic areareveals molecularly distinct populations of inhibitory and excitatoryneurons. Nat. Neurosci. 22, 642 (2019).

25. Hashimshony, T., Wagner, F., Sher, N. \& Yanai, I. CEL-Seq: single-cell RNA-Seq by multiplexed linear amplification. Cell Rep. 2, 666-73 (2012).

26. Wolf, F. A., Angerer, P. \& Theis, F. J. SCANPY: large-scale single-cell gene expression data analysis. Genome Biol. 19, 15 (2018).

27. Horvath, T. L., Bechmann, I., Naftolin, F., Kalra, S. P. \& Leranth, C. Heterogeneity in the neuropeptide Y-containing neurons of the rat arcuate nucleus: GABAergic and non-GABAergic subpopulations. Brain Res. 756, 283-286 (1997). 
28. Wittmann, G., Hrabovszky, E. \& Lechan, R. M. Distinct glutamatergic and GABAergic subsets of hypothalamic pro-opiomelanocortin neurons revealed by in situ hybridization in male rats and mice. J. Comp. Neurol. 521, 3287-3302 (2013).

29. Ciofi, P., Leroy, D. \& Tramu, G. Sexual dimorphism in the organization of the rat hypothalamic infundibular area. Neuroscience 141, 1731-1745 (2006).

30. Rupp, A. C. et al. Specific subpopulations of hypothalamic leptin receptor-expressing neurons mediate the effects of early developmental leptin receptor deletion on energy balance. Mol. Metab. 14, 130-138 (2018).

31. Burke, L. K. et al. Lorcaserin improves glycemic control via a melanocortin neurocircuit. Mol. Metab. 6, 1092-1102 (2017).

32. Berglund, E. D. et al. Serotonin 2C receptors in pro-opiomelanocortin neurons regulate energy and glucose homeostasis. J. Clin. Invest. 123, 5061-70 (2013).

33. Burke, L. K. et al. 5-HT obesity medication efficacy via POMC activation is maintained during aging. Endocrinology 155, 3732-8 (2014).

34. Frank, A., Brown, L. M. \& Clegg, D. J. The Role of Hypothalamic Estrogen Receptors in Metabolic Regulation. Front. Neuroendocrinol. 35, 550 (2014).

35. C, B., M, L., H, W., JN, W. \& T, H. Subtypes $Y 1$ and $Y 2$ of the Neuropeptide $Y$ Receptor Are Respectively Expressed in Pro-Opiomelanocortin- And neuropeptide-Y-containing Neurons of the Rat Hypothalamic Arcuate Nucleus. Neuroendocrinology 66, (1997).

36. Tannenbaum, G. S. Multiple Levels of Cross-Talk between Somatostatin (SRIF) and Growth Hormone (GH) -Releasing Factor in Genesis of Pulsatile GH Secretion. Clin. Pediatr. Endocrinol. 3, 97-110 (1994).

37. Tannenbaum, G. S., Gurd, W. \& Lapointe, M. Leptin Is a Potent Stimulator of Spontaneous Pulsatile Growth Hormone (GH) Secretion and the GH Response to GH-Releasing Hormone*. Endocrinology 139, 3871-3875 (1998).

38. E, C. et al. Role of Growth Hormone (GH)-releasing Hormone and Somatostatin on Leptin-Induced GH Secretion. Neuroendocrinology 69, (1999).

39. D, C., V, D. G. C., M, B., D, B. \& EE, M. Leptin Regulates GH Secretion in the Rat by Acting on GHRH and Somatostatinergic Functions. J. Endocrinol. 162, (1999). 
40. GS, T., WH, Z., M, L., P, Z. \& A, B. Growth Hormone-Releasing Hormone Neurons in the Arcuate Nucleus Express Both Sst1 and Sst2 Somatostatin Receptor Genes. Endocrinology 139, (1998).

41. Lazarus, M. et al. EP3 prostaglandin receptors in the median preoptic nucleus are critical for fever responses. Nat. Neurosci. 10, 1131-3 (2007).

42. Osaka, T. Heat loss responses and blockade of prostaglandin E2-induced thermogenesis elicited by alpha1-adrenergic activation in the rostromedial preoptic area. Neuroscience $162,1420-8$ (2009).

43. Yoshida, K. et al. Neurons of the rat preoptic area and the raphe pallidus nucleus innervating the brown adipose tissue express the prostaglandin E receptor subtype EP3. Eur. J. Neurosci. 18, 1848-1860 (2003).

44. Poon, K., Barson, J. R., Shi, H., Chang, G. Q. \& Leibowitz, S. F. Involvement of the CXCL12 System in the Stimulatory Effects of Prenatal Exposure to High-Fat Diet on Hypothalamic Orexigenic Peptides and Behavior in Offspring. Front. Behav. Neurosci. 11, 91 (2017).

45. Koch, M. Cannabinoid Receptor Signaling in Central Regulation of Feeding Behavior: A MiniReview. Front. Neurosci. 11, (2017).

46. $\mathrm{Xu}, \mathrm{B}$. et al. Brain-derived neurotrophic factor regulates energy balance downstream of melanocortin-4 receptor. Nat. Neurosci. 6, 736-42 (2003).

47. An, J. J., Liao, G.-Y., Kinney, C. E., Sahibzada, N. \& Xu, B. Discrete BDNF Neurons in the Paraventricular Hypothalamus Control Feeding andEnergy Expenditure. Cell Metab. 22, 175 (2015).

48. H, Y., JJ, A., C, S. \& B, X. Regulation of Energy Balance via BDNF Expressed in Nonparaventricular Hypothalamic Neurons. Mol. Endocrinol. 30, (2016).

49. Wang, H., Xie, H. \& Dey, S. K. Loss of Cannabinoid Receptor CB1 Induces Preterm Birth. PLoS One 3, e3320 (2008).

50. Kurrasch, D. M. et al. The neonatal ventromedial hypothalamus transcriptome reveals novel markers with spatially distinct patterning. J. Neurosci. 27, 13624-34 (2007).

51. Allison, M. B. et al. TRAP-seq defines markers for novel populations of hypothalamic and brainstem LepRb neurons. Mol. Metab. 4, 299-309 (2015).

52. Maguire, C. A. et al. Tac1 Signaling Is Required for Sexual Maturation and Responsiveness of GnRH 
Neurons to Kisspeptin in the Male Mouse. Endocrinology 158, 2319-2329 (2017).

53. Laque, A. et al. Leptin receptor neurons in the mouse hypothalamus are colocalized with the neuropeptide galanin and mediate anorexigenic leptin action. Am. J. Physiol. Endocrinol. Metab. 304, E999-1011 (2013).

54. Jall, S. et al. Pirt deficiency has subtle female-specific effects on energy and glucose metabolism in mice. Mol. Metab. 23, 75-81 (2019).

55. Wen, S., Aoki, M. \& Boehm, U. in Chemosensory Transduction: The Detection of Odors, Tastes, and Other Chemostimuli 339-357 (Elsevier Inc., 2016). doi:10.1016/B978-0-12-801694-7.00019-6

56. Dale, N. Purinergic signaling in hypothalamic tanycytes: Potential roles in chemosensing. Seminars in Cell and Developmental Biology 22, 237-244 (2011).

57. Langlet, F. Tanycyte Gene Expression Dynamics in the Regulation of Energy Homeostasis. Front. Endocrinol. (Lausanne). 10, 286 (2019).

58. H, H. et al. Evidence Indicating That Renal Tubular Metabolism of Leptin Is Mediated by Megalin but Not by the Leptin Receptors. Endocrinology 145, (2004).

59. Dietrich, M. O. et al. Megalin mediates the transport of leptin across the blood-CSF barrier. Neurobiol. Aging 29, 902-912 (2008).

60. Severi, l. et al. Activation of transcription factors STAT1 and STAT5 in the mouse median eminence after systemic ciliary neurotrophic factor administration. Brain Res. 1622, 217-29 (2015).

61. Vanlandewijck, M. et al. A molecular atlas of cell types and zonation in the brain vasculature. Nature 554, 475-480 (2018).

62. Kiefer, F. W. et al. Neutralization of osteopontin inhibits obesity-induced inflammation and insulin resistance. Diabetes 59, 935-46 (2010).

63. R, H., A, M., J, U., K, H. \& H, O. Effect of Leptin Administration on Myelination in ob/ob Mouse Cerebrum After Birth. Neuroreport 24, (2013).

64. Zhang, Y. et al. Purification and Characterization of Progenitor and Mature Human Astrocytes Reveals Transcriptional and Functional Differences with Mouse. Neuron 89, 37-53 (2016).

65. Lafrance, V., Inoue, W., Kan, B. \& Luheshi, G. N. Leptin modulates cell morphology and cytokine release in microglia. Brain. Behav. Immun. 24, 358-65 (2010). 
66. Lim, G., Wang, S., Zhang, Y., Tian, Y. \& Mao, J. Spinal leptin contributes to the pathogenesis of neuropathic pain in rodents. J. Clin. Invest. 119, 295-304 (2009).

67. Li, C. et al. SciBet as a portable and fast single cell type identifier. Nat. Commun. 11, 1818 (2020).

68. Aibar, S. et al. SCENIC: single-cell regulatory network inference and clustering. Nat. Methods 14, 1083-1086 (2017).

69. Van de Sande, B. et al. A scalable SCENIC workflow for single-cell gene regulatory network analysis. Nat. Protoc. 15, 2247-2276 (2020).

70. Lee, J. H. et al. Neuronal Deletion of Ghrelin Receptor Almost Completely Prevents Diet-Induced Obesity. Diabetes 65, 2169-78 (2016).

71. JZ, L. et al. Obesity and Metabolomics: Metallothioneins Protect Against High-Fat Diet-Induced Consequences in Metallothionein Knockout Mice. OMICS 19, (2015).

72. Guo, T. et al. Adipocyte ALK7 links nutrient overload to catecholamine resistance in obesity. Elife 3, e03245 (2014).

73. N, S. et al. A Role for VGF in the Hypothalamic Arcuate and Paraventricular Nuclei in the Control of Energy Homeostasis. Neuroscience 265, (2014).

74. Cady, G. et al. Hypothalamic growth hormone receptor (GHR) controls hepatic glucose production in nutrient-sensing leptin receptor (LepRb) expressing neurons. Mol. Metab. 6, 393-405 (2017).

75. MO, G. et al. SORCS1: A Novel Human Type 2 Diabetes Susceptibility Gene Suggested by the Mouse. Diabetes 56, (2007).

76. A, S. et al. SORCS1 and SORCS3 Control Energy Balance and Orexigenic Peptide Production. EMBO Rep. 19, (2018).

77. K, H., T, V. \& J, V. TRPM3 in Temperature Sensing and Beyond. Temp. (Austin, Tex.) 2, (2015).

78. W, S. et al. Activation of TRPV2 Negatively Regulates the Differentiation of Mouse Brown Adipocytes. Pflugers Arch. 468, (2016).

79. van der Klaauw, A. A. et al. Human Semaphorin 3 Variants Link Melanocortin Circuit Development and Energy Balance. Cell 176, 729-742.e18 (2019).

80. LN, Z. et al. Beta-arrestin-1 Protein Represses Diet-Induced Obesity. J. Biol. Chem. 286, (2011).

81. VAGENA, E. et al. 1713-P: ASB4 Regulates Glucose Homeostasis and Body Adiposity during Aging. 
Diabetes 68, 1713-P (2019).

82. Stechschulte, L. A. et al. FKBP51 Controls Cellular Adipogenesis through p38 Kinase-Mediated Phosphorylation of GR $\alpha$ and PPARY. Mol. Endocrinol. 28, 1265 (2014).

83. M, F. et al. FKBP5 Polymorphism Is Associated With Insulin Resistance in Children and Adolescents With Obesity. Obes. Res. Clin. Pract. 12, (2018).

84. Gómez-Serrano, M. et al. Proteome-wide alterations on adipose tissue from obese patients as age-, diabetes- and gender-specific hallmarks. Sci. Rep. 6, 25756 (2016).

85. Gao, S., Howard, S. \& LoGrasso, P. V. Pharmacological Inhibition of c-Jun N-terminal Kinase Reduces Food Intake and Sensitizes Leptin's Anorectic Signaling Actions. Sci. Rep. 7, 41795 (2017).

86. Abdelli, S. \& Bonny, C. JNK3 maintains expression of the insulin receptor substrate 2 (IRS2) in insulin-secreting cells: functional consequences for insulin signaling. PLoS One 7, e35997 (2012).

87. Feng, J. et al. The Role of JNk Signaling Pathway in Obesity-Driven Insulin Resistance. Diabetes. Metab. Syndr. Obes. 13, 1399-1406 (2020).

88. Solinas, G. \& Becattini, B. JNK at the crossroad of obesity, insulin resistance, and cell stress response. Mol. Metab. 6, 174-184 (2017).

89. Vernia, S. et al. Excitatory transmission onto AgRP neurons is regulated by cJun NH2-terminal kinase 3 in response to metabolic stress. Elife 5, e10031 (2016).

90. An, J. J. et al. TrkB-expressing paraventricular hypothalamic neurons suppress appetite through multiple neurocircuits. Nat. Commun. 11, 1729 (2020).

91. Liao, G.-Y., Kinney, C. E., An, J. J. \& Xu, B. TrkB-expressing neurons in the dorsomedial hypothalamus are necessary and sufficient to suppress homeostatic feeding. Proc. Natl. Acad. Sci. U. S. A. 116, 3256-3261 (2019).

92. Ozek, C., Zimmer, D. J., De Jonghe, B. C., Kalb, R. G. \& Bence, K. K. Ablation of intact hypothalamic and/or hindbrain TrkB signaling leads to perturbations in energy balance. Mol. Metab. 4, 867-880 (2015).

93. Tsao, D. et al. TrkB agonists ameliorate obesity and associated metabolic conditions in mice. Endocrinology 149, 1038-48 (2008).

94. Zeeni, N. et al. A positive change in energy balance modulates TrkB expression in the hypothalamus and nodose ganglia of rats. Brain Res. 1289, 49-55 (2009). 
95. Kim, S.-J. et al. Neural regulation of energy and bone homeostasis by the synaptic adhesion molecule Calsyntenin-3. Exp. Mol. Med. 52, 793-803 (2020).

96. Zeng, X. et al. Innervation of thermogenic adipose tissue via a calsyntenin 3 $\beta-\mathrm{S} 100 \mathrm{~b}$ axis. Nature 569, 229-235 (2019).

97. Chen, S. et al. Predicted secreted protein analysis reveals synaptogenic function of Clstn3 during WAT browning and BAT activation in mice. Acta Pharmacol. Sin. 40, 999-1009 (2019).

98. Thorleifsson, G. et al. Genome-wide association yields new sequence variants at seven loci that associate with measures of obesity. Nat. Genet. 41, 18-24 (2009).

99. Wu, L. et al. Influence of lifestyle on the FAIM2 promoter methylation between obese and lean children: a cohort study. BMJ Open 5, e007670 (2015).

100. Boender, A. J., Van Rozen, A. J. \& Adan, R. A. H. Nutritional State Affects the Expression of the Obesity-Associated Genes Etv5, Faim2, Fto , and Negr1. Obesity 20, 2420-2425 (2012).

101. Timshel, P. N., Thompson, J. J. \& Pers, T. H. Mapping heritability of obesity by brain cell types. bioRxiv 2020.01.27.920033 (2020). doi:10.1101/2020.01.27.920033

102. Loh, P.-R., Kichaev, G., Gazal, S., Schoech, A. P. \& Price, A. L. Mixed-model association for biobankscale datasets. Nat. Genet. 50, 906-908 (2018).

103. Komori, T., Morikawa, Y., Nanjo, K. \& Senba, E. Induction of brain-derived neurotrophic factor by leptin in the ventromedial hypothalamus. Neuroscience 139, 1107-15 (2006).

104. Liao, G.-Y. et al. Dendritically targeted Bdnf mRNA is essential for energy balance and response to leptin. Nat. Med. 18, 564-71 (2012).

105. Chao, H., Digruccio, M., Chen, P. \& Li, C. Type 2 corticotropin-releasing factor receptor in the ventromedial nucleus of hypothalamus is critical in regulating feeding and lipid metabolism in white adipose tissue. Endocrinology 153, 166-76 (2012).

106. Muraro, M. J. et al. A Single-Cell Transcriptome Atlas of the Human Pancreas. Cell Syst. 3, 385394.e3 (2016).

107. Krueger, F. TrimGalore. github (2020). at <https://github.com/FelixKrueger/TrimGalore/>

108. Martin, M. Cutadapt removes adapter sequences from high-throughput sequencing reads. EMBnet.journal 17, 10 (2011).

109. Simon Andrews. Babraham Bioinformatics - FastQC A Quality Control tool for High Throughput 
Sequence Data. at <http://www.bioinformatics.babraham.ac.uk/projects/fastqc/>

110. Dobin, A. \& Gingeras, T. R. Mapping RNA-seq Reads with STAR. Curr. Protoc. Bioinforma. 51, 11.14.1-11.14.19 (2015).

111. Traag, V. A., Waltman, L. \& van Eck, N. J. From Louvain to Leiden: guaranteeing well-connected communities. Sci. Rep. 9, 5233 (2019).

112. arboreto. at <https://arboreto.readthedocs.io/en/latest/>

113. Pers, T. H. CELLECT. github at <https://github.com/perslab/CELLECT>

114. Pers, T. H. CELLEX. github at <https://github.com/perslab/CELLEX>

115. Demontis, D. et al. Discovery of the first genome-wide significant risk loci for ADHD. bioRxiv 145581 (2017). doi:10.1101/145581

116. Duncan, L. et al. Significant Locus and Metabolic Genetic Correlations Revealed in Genome-Wide Association Study of Anorexia Nervosa. Am. J. Psychiatry 174, 850-858 (2017).

117. Pulit, S. L. et al. Meta-analysis of genome-wide association studies for body fat distribution in 694649 individuals of European ancestry. Hum. Mol. Genet. 28, 166-174 (2019).

118. Yengo, L. et al. Meta-analysis of genome-wide association studies for height and body mass index in 700000 individuals of European ancestry. Hum. Mol. Genet. 27, 3641-3649 (2018).

119. Pardiñas, A. F. et al. Common schizophrenia alleles are enriched in mutation-intolerant genes and in regions under strong background selection. Nat. Genet. 50, 381-389 (2018).

120. Bradfield, J. P. et al. A genome-wide meta-analysis of six type 1 diabetes cohorts identifies multiple associated loci. PLoS Genet. 7, e1002293 (2011).

121. Mahajan, A. et al. Fine-mapping type 2 diabetes loci to single-variant resolution using high-density imputation and islet-specific epigenome maps. Nat. Genet. 50, 1505-1513 (2018).

122. Xue, A. et al. Genome-wide association analyses identify 143 risk variants and putative regulatory mechanisms for type 2 diabetes. Nat. Commun. 9, 2941 (2018).

123. Nagel, M. et al. Meta-analysis of genome-wide association studies for neuroticism in 449,484 individuals identifies novel genetic loci and pathways. Nat. Genet. 50, 920-927 (2018).

124. Franklin, K. B. J. \& Paxinos, G. Paxinos and Franklin's The mouse brain in stereotaxic coordinates. 